The Astrophysical Journal, submitted

Preprint typeset using LATEX style emulateapj v. 25/04/01

\title{
STATISTICS OF MAGNIFICATION PERTURBATIONS BY SUBSTRUCTURE IN THE COLD DARK MATTER COSMOLOGICAL MODEL
}

\author{
Eduardo Rozo ${ }^{1,2}$, Andrew R. Zentner ${ }^{2,3}$, Gianfranco Bertone ${ }^{4}$, And Jacqueline Chen ${ }^{2,3}$ \\ The Astrophysical Journal, submitted
}

\begin{abstract}
We study the statistical properties of magnification perturbations by substructures in strong lensed systems using linear perturbation theory and an analytical substructure model including tidal truncation and a continuous substructure mass spectrum. We demonstrate that magnification perturbations are dominated by perturbers found within roughly a tidal radius of an image, and that sizable magnification perturbations may arise from small, coherent contributions from several substructures within the lens halo. The root-mean-square (rms) fluctuation of the magnification perturbation is $\sim 10 \%$ to $\sim 20 \%$ and both the average and rms perturbations are sensitive to the mass spectrum and density profile of the perturbers. Interestingly, we find that relative to a smooth model of the same mass, the average magnification in clumpy models is lower (higher) than that in smooth models for positive (negative) parity images. This is opposite from what is observed if one assumes that the image magnification predicted by the best-fit smooth model of a lens is a good proxy for what the observed magnification would have been if substructures were absent. While it is possible for this discrepancy to be resolved via nonlinear perturbers, we argue that a more likely explanation is that the assumption that the best-fit lens model is a good proxy for the magnification in the absence of substructure is not correct. We conclude that a better theoretical understanding of the predicted statistical properties of magnification perturbations by CDM substructure is needed in order to affirm that CDM substructures have been unambiguously detected.
\end{abstract}

Subject headings: cosmology: theory - dark matter - galaxies: formation, halos, structure

\section{INTRODUCTION}

\subsection{Evidence for Small-Scale Features in Strong Lenses}

Standard lens models often have difficulties explaining the relative fluxes of multiply-imaged sources (Metcalf \& Zhao 2002). Perhaps the simplest explanation for these discrepancies is that they are electromagnetic in origin. Observed fluxes may be affected by obscuration due to dust, scintillation in the galaxy, or some other form of electromagnetic phenomena (e.g., Koopmans 2003). However, there is strong evidence that these are not the only source of the discrepancies. In particular, Kochanek \& Dalal (2004) have shown that positive-parity images in observed lenses tend to be brighter than what the standard lens models predict, while negative-parity images tend to be demagnified. Electromagnetic effects do not distinguish between positive-parity and negative-parity images, so we are left to conclude that flux anomalies must be produced by gravity. That is, the lensing potentials of real galaxies are not fully characterized by the simple models used to describe them.

The next simplest explanation for these discrepancies is that standard lens models are either overly simplistic or overly restrictive (Kawano 2004; Evans \& Witt 2003, though see also Yoo et al. 2005). For instance, environmental effects are often modeled as a constant, external shear, whereas detailed modeling of the lens environment might be necessary (Möller 2002; Keeton \& Zabludoff 2004). Again, there is strong evidence that this is not the only difficulty.

The first piece of evidence that other difficulties must exist concerns the so-called cusp relation. Cusp lens configurations occur when a source is located near one of the cusps of a lens'

\footnotetext{
${ }^{1}$ Department of Physics, The University of Chicago, Chicago, IL 60637, USA erozododdjob.uchicago.edu

${ }^{2}$ Kavli Institute for Cosmological Physics, Chicago, IL 60637, USA

3 Department of Astronomy and Astrophysics, The University of Chicago, Chicago, IL 60637, USA

4 NASA/Fermilab Astrophysics Center, Fermi National Accelerator Laboratory, Batavia, IL 60510, USA
}

tangential caustic. They characteristically have a tight cluster of three images and a significantly more isolated fourth image. ${ }^{5}$ The cusp relation relates the signed fluxes $\Gamma_{i}$, of each of the images (labeled by index i), where the sign of the flux is given by the parity of the image. For cusp configurations, one can define the cusp parameter $R=\sum_{\mathrm{i}} \Gamma_{\mathrm{i}} /\left(\sum_{\mathrm{i}}\left|\Gamma_{\mathrm{i}}\right|\right)$, where the sum is over the three tightly-clustered images. For a point source located near the cusp, one expects to find $R \approx 0$ so long as the lensing potential is featureless on scales comparable to or smaller than the typical image separations (Schneider \& Weiss 1992; Zakharov 1995; Gaudi \& Petters 2002). Keeton et al. (2003) have shown that careful analysis can identify lenses where the condition $R \approx 0$ is violated, providing strong evidence that smallscale features in the lensing potential are present. A similar argument can be made for fold lenses where one expects a close pair of images with equal and opposite fluxes in addition to two isolated images. This fold relation is likewise violated in a large number of lenses (Keeton et al. 2005). Note that while such detections are model independent and robust, they require further model-dependent analysis to determine possible causes of the perturbation and to identify which image (or images) is (are) perturbed (e.g., Mao \& Schneider 1998; Keeton 2001; Dobler $\&$ Keeton 2005).

A second piece of evidence for small-scale structure in lenses is the frequency dependence of the flux ratios of multiplyimaged sources (Moustakas \& Metcalf 2003). This argument hinges on the fact that features in the lensing potential smaller than the source size tend to be smoothed out by the source. As an illustration, consider two spatially-coincident sources of known brightness, one large and one small, lensed by an intervening lens potential with features on scales intermediate between the two source sizes. In such a case, the smaller source is strongly affected by these intermediate features, whereas the larger source is not because the observed image flux constitutes

5 A fourth image may be absent in the so-called "naked cusp" lenses, which occur when the cusp of a tangential caustic is not contained in the region interior to the radial caustic in the plane of the source. 
an average over a region larger than the scale of the features. Therefore, the existence of the features is signaled through different observed magnifications for each of the two sources. Fortunately, Nature has been kind enough to provide exactly this kind of setup. In particular, the spatial extent of the emission region of a quasar depends on the particular frequency at which the quasar is observed (e.g., Kembhavi \& Narlikar 1999). By comparing flux ratios of multiply-imaged quasars in different spectral regions, one may search for structure in the lens potential on length scales intermediate between those of the quasar emission regions. Again, more detailed modeling is necessary to determine the nature of the perturbation (Metcalf 2004).

\subsection{CDM Substructure as a Possible Source for Small-Scale Features in the Lensing Potential}

To our knowledge, Mao \& Schneider (1998) were the first to propose that intermediate-mass-scale substructures within a lens could explain the problem posed by anomalous flux ratios. The Cold Dark Matter (CDM) paradigm of cosmological structure formation (e.g., Blumenthal et al. 1984; White \& Rees 1978) predicts just such substructure. In recent years, a new generation of numerical simulations of structure formation in the CDM paradigm revealed that the dark matter halos that are believed to host galaxies generally have $\sim 10 \%$ of their mass in distinct, gravitationally-bound, substructures commonly referred to as subhalos or satellite halos (e.g., Klypin et al. 1999; Moore et al. 1999; Ghigna et al. 2000; De Lucia et al. 2004; Diemand et al. 2004; Gao et al. 2004), and it was quickly realized that these subhalos are an ideal candidate for the source of the lensing perturbations (see Moore et al. 1999). Conversely, estimates of the abundance of substructure can serve as a test of models of cosmological structure formation (e.g., Dalal \& Kochanek 2002b; Zentner \& Bullock 2003).

The amount of substructure predicted by the CDM model has been the subject of numerous recent studies. One apparent discrepancy between theory and observation is that there are more than an order of magnitude fewer dwarf satellite galaxies about the Milky Way and M31 than the number of subhalos of comparable velocity dispersion predicted by the CDM paradigm (Kauffmann et al. 1993; Klypin et al. 1999; Moore et al. 1999). This mismatch is known as the "missing satellites problem," and many possible resolutions have been considered. Several authors have proposed modifications to the properties of the dark matter, making it "warm" rather than cold (Hogan \& Dalcanton 2000; Colín et al. 2000; Bode et al. 2001; Lin et al. 2001; Knebe et al. 2002) or introducing a self interaction for the dark matter (Spergel \& Steinhardt 2000) in order to reduce the amount of substructure.

Alternatively, any suppression in the amount of small-scale power in the primordial spectrum of density fluctuations can significantly influence halo structure (Zentner \& Bullock 2002; McGaugh et al. 2003; van den Bosch et al. 2003) and may reduce the abundance of subhalos (e.g., Kamionkowski \& Liddle 2000; Sigurdson \& Kamionkowski 2004). Zentner \& Bullock (2003) studied in detail the dependence of halo substructure on the primordial power spectrum and showed that even mild modifications to the power spectrum on small scales can greatly affect the severity and interpretation of the missing satellites problem. These authors also estimated projected subhalo mass fractions for several different models, demonstrating how measurements of this quantity through lensing flux anomalies (e.g., Dalal \& Kochanek 2002a) could provide information about the nature of the dark matter and the primordial power spectrum, and thus inflation, on scales that may be otherwise inaccessible.

The most conservative solution to the missing satellites problem is that star formation may be naturally suppressed in a large fraction of small halos due to feedback from supernovae (e.g., Dekel \& Silk 1986; Kauffmann et al. 1993; Cole et al. 1994; Somerville \& Primack 1999) or from the ionizing background (e.g., Rees 1986; Efstathiou 1992; Kauffmann et al. 1993; Shapiro et al. 1994; Thoul \& Weinberg 1996; Bullock et al. 2000; Somerville 2002; Benson et al. 2002). This leads to two possible scenarios. The feedback mechanism could cause an abrupt drop in the efficiency of galaxy formation in small halos, in which case the MW satellites should lie in the eleven most massive subhalos surrounding the MW. This could be true if the mapping between observed stellar velocity dispersions of the satellites and the size of the subhalos that host these satellites allows for the satellites to sit in large subhalos due to the significant tidal evolution of the subhalos (e.g. Stoehr et al. 2002; Hayashi et al. 2003, though recent work by Kazantzidis et al. 2004b does not support this scenario). Contrarily, feedback could set in gradually so that small subhalos become increasingly less likely to host luminous satellites. Including several other details, this type of scenario leads to a model of biased galaxy formation in small halos that appears to agree well with many observed features of the MW dwarfs (Kravtsov et al. 2004; Zentner et al. 2005c).

If inefficient galaxy formation in small halos is the resolution to the missing satellites problem, galaxy-sized subhalos should be filled with massive, dark subhalos that would be devoid of stars and detectable only through their gravitational influence or, more speculatively, through the detection of gamma-rays (e.g., Silk \& Stebbins 1993; Berezinsky et al. 1997; Bergström et al. 1999; Calcáneo-Roldán \& Moore 2000; Baltz et al. 2000; Tasitsiomi \& Olinto 2002; Stoehr et al. 2003; Koushiappas et al. 2004) or antiprotons (e.g., Bergström et al. 1999; Bottino et al. 1998; Donato et al. 2004) from dark matter annihilations in their dense cores (for further details see Bertone et al. (2005) and references therein). An unequivocal detection of subhalos through lensing could be used to constrain the properties of the dark matter and the primordial power spectrum on small scales (Dalal \& Kochanek 2002b; Zentner et al. 2005b). A detection at sufficiently high levels could represent an enormous triumph for the CDM paradigm of structure formation with a standard, scale-invariant primordial power spectrum. Such a detection could help determine the nature of the feedback mechanism that leads to the dearth of small, luminous satellites in the MW and distinguish between the proposal of Stoehr et al. (2002) and Hayashi et al. (2003) and that of Kravtsov et al. (2004).

It is important to note that, while dark matter substructure within the lens halo seems to be the leading interpretation of observed flux anomalies, it is certainly not the only possibility. Other possible sources of small-scale fluctuations in lens potentials include stars, correlated and uncorrelated external halos along the line of sight (Chen et al. 2003b; Möller \& Blain 2001; Metcalf 2004a,b; Wambsganss et al. 2004), and possible disk structures within the galaxy (Quadri et al. 2003; Möller et al. 2003; Bradăc et al. 2004; Amara et al. 2004). Microlensing by stars in the lens galaxy, while expected and observed in some cases (e.g., Schild 1996; Refsdal 2000; Wozniak 2000; Wisotzki 2003; Schechter 2003; Richards 2004), should not affect the radio or narrow-line emission fluxes due to the source 
size (Moustakas \& Metcalf 2003; Kochanek \& Dalal 2004; Dobler \& Keeton 2005), and cannot be the cause of radio flux anomalies.

In light of this discussion, predicting the properties of magnification perturbations due to CDM substructures in realistic lens systems is of paramount importance. Unfortunately, the detailed CDM predictions for lens systems are not yet completely understood. The first issue to address is whether or not the amount of substructure predicted by the standard CDM model is consistent with observed flux perturbations. This problem has been addressed analytically (Chen et al. 2003b), numerically (Metcalf \& Madau 2001), and observationally (Dalal \& Kochanek 2002b; Metcalf \& Zhao 2002; Chiba 2002; Bradăc 2002) with fairly consistent results: a projected substructure mass fraction, $f_{\text {sub }} \equiv \Sigma_{\text {sub }} / \Sigma_{\text {tot }}$, of order a few percent near the images can reproduce the flux perturbations in observed gravitational lenses. Whether this constitutes agreement with CDM or not is unclear. While the total mass fraction in substructure for CDM halos is $\sim 10 \%$, naive theoretical estimates of the projected subhalo mass fraction near typical Einstein radii of lenses ( $\sim$ a few percent of the halo virial radius) are significantly lower, yielding $f_{\text {sub }} \lesssim 0.5 \%$ (Zentner \& Bullock 2003; Mao et al. 2004). However, it is not clear whether such a naïve comparison is fair (Chen \& Rozo 2005, in preparation) because massive substructures could have important effects even if they do not fall within the Einstein radius of the host galaxy. Further, spatially-biased substructure distributions will clearly affect the lensing potential, and to what extent these effects can be subsumed within the lens model remains unknown. In related investigations, Bradǎc et al. (2004) have argued that the predicted amount of substructure in lenses is sufficient to explain the level of cusp relation violations observed in the data using simulations of galaxy formation in CDM, though Amara et al. (2004) (see also Macció et al. 2005) reached the opposite conclusion. In summary, strong lensing is an important probe of small-scale structure and the question of whether magnification perturbations are observed at the level that is expected from cold dark matter substructures has not been answered unambiguously.

\subsection{This Work}

In the present work, we quantify the magnifications perturbations predicted by the CDM model of structure formation and develop an understanding of the important aspects to consider highlighting several key parameters. In particular, we predict the statistical properties of magnification perturbations due to subhalos in realistic lens models. We begin in $\S 2$ by generalizing the work of Keeton (2003). In particular, using linear perturbation theory we derive a general expression for the cross section of a single perturber to produce a magnification perturbation above some given size assuming only circularlysymmetric and monotonically-decreasing substructure surface density profiles. Appendix B and Appendix C illustrate our formalism in the context of point mass and Singular Isothermal Sphere (SIS) perturbers. In this context, we highlight the importance of tidal truncation on the probability of finding nonlinear perturbers by showing that the expected number of nonlinear perturbers increases by an order of magnitude if the tidal truncation of substructure is ignored.

In $\S 3$ we build on these results to derive expressions for the average and variance of the total magnification perturbation due to an ensemble of perturbers (not necessarily identical).
We then apply our results to the specific problem of magnification perturbations by CDM substructures within lens galaxies in $\S 4$. We use a simple, yet realistic, model for halo substructure which includes tidal truncation and a substructure mass spectrum, and thereby predict the average and variance of the magnification perturbations generated by CDM substructures. This type of argument provides an important statistical test of the CDM paradigm, which is particularly relevant given that the level of substructure near the Einstein radius of CDM halos is expected to vary enormously from halo to halo (e.g., Zentner \& Bullock 2003; Mao et al. 2004; Taylor \& Babul 2005; Zentner et al. 2005a). Furthermore, our analytical treatment allows us to identify and isolate the various characteristic of substructures to which the statistical properties of magnification perturbations are sensitive to, such as substructure profiles and their mass spectrum.

We find that small, negative values for the average magnification perturbation are a generic prediction. In other words, positive-parity images generated by a lens with substructure are dimmer than the corresponding images for a lens of the same mass with no substructure. We show that this can be understood as a byproduct of requiring perturbations to be linear: introduction of a nonlinear cutoff implies that a small fraction of the mass is ignored, which leads to a small negative magnification perturbation. The dimming of positive parity images is opposite to what is observed if one assumes that the best-fit model for a lens is a good proxy for the lensing potential obtained by replacing all substructures by a smooth component, which we argue is likely not a good assumption. In $\S 5$ we summarize our results and draw conclusions. Lastly, in Appendix A, we discuss in detail the validity of linear perturbation theory, and argue that linear perturbation theory should be valid whenever astrometric perturbations are negligible. Finally Appendix B and Appendix $\mathrm{C}$ illustrate our algorithm for computing magnification perturbation cross sections explicitly in the cases of point mass and singular isothermal sphere perturbers respectively.

\section{MAGNIFICATION PERTURBATION CROSS SECTIONS}

In this section, we illustrate a general algorithm for computing the cross sections $\sigma(\delta)$ for individual perturbers to generate magnification perturbations of size $\delta \equiv \delta \mu /|\mu|$ or larger ( $\mu$ is the unperturbed image magnification). Note that for a constant surface number density of perturbers $s$, the probability of finding a perturber that creates a perturbation of size $\delta$ is simply $d P=s|d \sigma / d \delta| d \delta$. This forms the basis of our analysis of multiple perturbers and underlines the importance of understanding the magnification perturbation cross section $\sigma(\delta)$.

Consider the image of a point source lensed by a smooth, projected lensing potential $\psi$, which we refer to as the macrolens or macromodel, and let $\kappa$ and $\gamma$ be the values of the convergence and shear fields at the image position. We investigate the magnification perturbation of the image when one introduces a small perturbation to the potential, $\delta \psi$. In general, the perturbation $\delta \psi$ will not only change the flux of the image, but also its position. However, here we will work in the limit that astrometric perturbations are negligible. We emphasize that negligible astrometric perturbations means that the change in the convergence and shear values of both the macrolens and the perturber have to be negligible. As we show in Appendix A, we expect this assumption to hold for small flux perturbations, 
that is, when

$$
|\delta|=\left|\frac{\delta \mu}{\mu}\right| \ll 1
$$

where the flux perturbation $\delta \mu$ is evaluated at the unperturbed image position. Consequently, we will only be considering small perturbations to the flux.

Let $\delta \psi, \delta \kappa$, and $-\delta \gamma(\cos (2 \phi), \sin (2 \phi))$ be the profiles of the potential, convergence, and the two components of the shear of an individual perturber. We assume that all profiles vary monotonically. The Poisson equation for the potential $\psi$ is linear, so the total convergence and shear are given simply by $\kappa+\delta \kappa$ and $(\gamma-\delta \gamma \cos (2 \phi),-\delta \gamma \sin (2 \phi))$. Linearizing the magnification $\mu^{-1}=(1-\kappa)^{2}-\gamma^{2}$, results in 6

$$
\delta(\theta, \phi)=\frac{\delta \mu}{|\mu|}=2|\mu|[(1-\kappa) \delta \kappa(\theta)-\gamma \delta \gamma(\theta) \cos (2 \phi)],
$$

where $\kappa$ and $\gamma$ are the convergence and shear of the macromodel respectively. We have taken the origin to be at the center of the perturber, and the coordinates $\theta$ and $\phi$ represent twodimensional polar coordinates. In what follows, it is more convenient to choose the image (assumed fixed) as the origin of the coordinate system. In this case, the angle $\theta$ is still the separation between the image and the perturber, but $\phi$ becomes the angle of the perturber's position. One may check that the above expression remains valid. The perturbation $\delta$ as a function of perturber position is illustrated in figure 1. Using Equation (2), we define the cross section for magnification perturbations stronger than $\Delta>0$ as

$$
\sigma(\Delta)=\int_{\delta>\Delta}(\theta d \theta d \phi)=\int(\theta d \theta d \phi) \mathcal{H}(\delta-\Delta),
$$

where $\mathcal{H}(x)$ is the Heaviside step function. Of course, if one is interested in perturbations $\delta<0$, one ought to integrate over the region $\delta<\Delta$ instead. $^{7}$ To carry out the integral, one might fix the angle $\phi$, find the radial region over which perturbations stronger than $\Delta$ exist, and then integrate over all angles. Unfortunately, this is not possible in general because the equation $\delta(\theta, \phi)=\Delta$ defines the $\theta$-coordinate boundary implicitly. A better approach is to first find the range of radii $\theta \in\left[\theta_{\min }, \theta_{\max }\right]$, over which perturbations of size $\Delta$ or stronger are possible. Next, for all radii within this interval, find the angular region over which perturbers produce perturbations stronger than $\Delta$. Then lastly, integrate this angular region over all radii to obtain the cross section. The advantage here is that the simple $\phi$ dependence of the perturbation $\delta(\theta, \phi)$ allows one to solve explicitly for the relevant angular intervals.

Consider Equation (2). As $\theta \rightarrow \infty$, we have $\delta \rightarrow 0$, so it follows that if perturbations ever get stronger than $\Delta$, there must be a maximum distance $\theta_{\max }(\Delta)$ at which $\delta=\Delta$ first occurs. The quantity $\delta(\theta, \phi)$ is always bounded by $2|\mu|((1-\kappa) \delta \kappa \pm$ $\gamma \delta \gamma)$, so $\theta_{\max }(\Delta)$ is given by the solution to

$$
\theta_{\max }(\Delta)= \begin{cases}2|\mu|\left[(1-\kappa) \delta \kappa\left(\theta_{\max }\right)+\gamma \delta \gamma\left(\theta_{\max }\right)\right] & \text { if } \Delta>0 \\ 2|\mu|\left[(1-\kappa) \delta \kappa\left(\theta_{\max }\right)-\gamma \delta \gamma\left(\theta_{\max }\right)\right] & \text { if } \Delta<0\end{cases}
$$

If perturbations stronger than $\Delta$ are never produced, we set $\theta_{\text {max }}(\Delta)=0$. It is important to note that even though both $\delta \kappa$

6 The negative sign in front of $\gamma$ in Equation (2) is due to our sign convention. One has $\vec{\gamma}=-\gamma(\cos (2 \phi), \sin (2 \phi))$, so aligning the shear with the $x$ axis can mean either $\phi=0$ for $\vec{\gamma}=(-\gamma, 0)$ or $\phi=\pi / 2$ for $\vec{\gamma}=(\gamma, 0)$. We use the latter.

7 We rely on context to specify whether the phrase "perturbations stronger than $\Delta$ " means $|\delta|>\Delta$ regardless of the sign of $\delta$, or $\delta<\Delta$ for negative $\Delta$ and $\delta>\Delta$ for positive $\Delta$. For instance, in this section we will be mostly interested in the latter case, but when computing the number of nonlinear perturbers, one is interested in perturbers with $|\delta| \gtrsim \Delta \approx 1$ regardless of the sign of $\delta$.

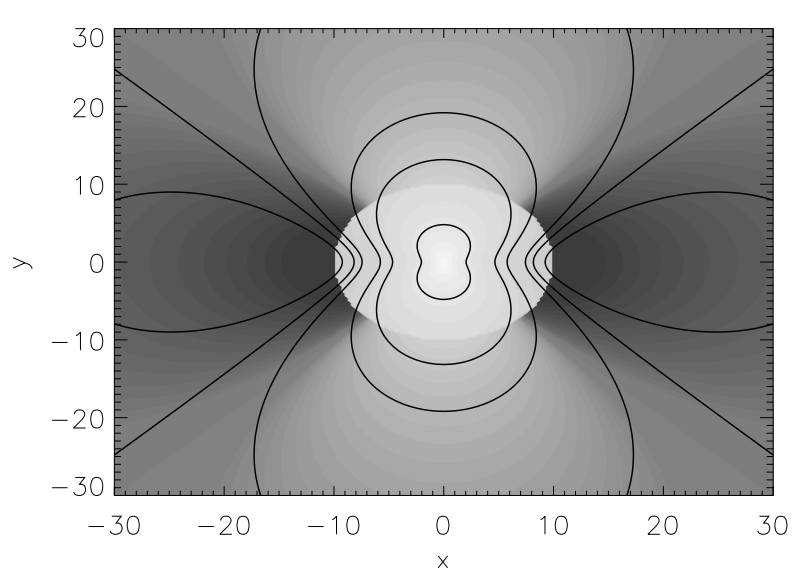

FIG. 1.- Shown above are the $\delta$ contours around a positive parity image. The field $\delta(\mathbf{x})$ is defined as the size of the magnification perturbation experienced by an image at the origin when a perturber is placed at point $\mathbf{x}$. The shaded contours are obtained assuming truncated SIS perturbers, with dark being negative and light being positive. The boundaries extending out at $45^{\circ}$ angles in the shaded contours correspond to $\delta=0$ (no perturbation). Distances are measured in units of the Einstein radius and the tidal radius is assumed to be ten times the Einstein radius, clearly seen as a discontinuity in the shaded contours. The angle $\phi$ is defined relative to the $x$ axis, itself defined such that the macroshear is $\gamma=(\gamma, 0)$. The solid contours are obtained using a PseudoGaffe profile, and correspond to $\delta=\{-0.01,0.0,0.01,0.05,0.10,0.50\}$. The double lobe features of SIS perturbers found by Keeton (2003) are evident at distances smaller than the tidal radius.

and $\delta \gamma$ are monotonic by assumption, $\delta(\theta, \phi)$ need not be. If this is the case Equation (4), may have two roots: $\theta_{\max }(\Delta)$ is defined as the larger of the two roots, while the second root is the minimum radius $\theta_{\min }(\Delta)$, for which perturbations stronger than $\Delta$ exist. If Eq. (4) has only one or no roots, we set $\theta_{\min }(\Delta)=0$. One might expect perturbations always to become stronger as the perturber approaches the image (i.e. that $\delta$ varies monotonically), but this is not necessarily so. As a counterexample, point masses may create negative perturbations on a positiveparity image, while singular isothermal sphere (SIS) perturbers cannot. Consider an SIS truncated at some finite radius and placed at infinity so as to produce a negative perturbation. As $\theta$ decreases, the perturbation grows more negative, until the distance from the image is equal to the truncation radius. At that point, the perturbation becomes positive. A truncated SIS has a minimum radius for which negative perturbations are possible.

Let us find the angular region over which perturbations stronger than $\Delta$ exist for a fixed radius $\theta$. The boundary points are defined by $\delta(\theta, \phi)=\Delta$, with a solution for $\phi \in[0, \pi / 2]$ given by $^{8}$

$$
\phi_{\mathrm{m}}(\theta \mid \Delta)=\frac{1}{2} \cos ^{-1}\left\{\frac{1}{\gamma \delta \gamma(\theta)}\left[(1-\kappa) \delta \kappa(\theta)-\frac{\Delta}{2|\mu|}\right]\right\} .
$$

Of course, a second solution, $\phi=\pi-\phi_{\mathrm{m}}$, exists in the region $\phi \in[\pi / 2, \pi]$. In principle, one is also interested in solutions with $\phi \in[\pi, 2 \pi]$, but perturbations are symmetric under reflection about the axis $\phi=0$, so the total cross section is double that obtained by integrating Eq. (3) over the region $\phi \in[0, \pi]$. Finally, we can check that $\delta$ always increases with $\phi$ from $\phi=\phi_{\mathrm{m}}$ while $\delta$ decreases with $\phi$ from $\phi=\pi-\phi_{\mathrm{m}}$. The relevant angular interval for $\delta>\Delta$ with $\Delta>0$ is thus $\phi \in\left[\phi_{m}, \pi-\phi_{m}\right]$, whereas

${ }^{8}$ It is this step that is not always possible to perform when trying to determine the allowed radial region for a fixed angle $\phi$. However, when possible, switching the order of integration may prove simpler (for example, see Keeton 2003). 
for $\Delta<0$, the appropriate region is $\phi \in\left[0, \phi_{\mathrm{m}}\right] \cup\left[\pi-\phi_{\mathrm{m}}, \pi\right]$. Physically, this statement says that perturbers are most effective at brightening positive-parity images when placed perpendicular to the shear axis $\gamma_{1}=\gamma$, while the opposite is true of negative-parity images.

One possibility we have not considered is that there is no solution to $\delta(\theta, \phi)=\Delta$. For $\theta_{\min }<\theta<\theta_{\max }$, one is guaranteed to have perturbations at least as strong as $\Delta$, so a lack of solutions implies that at the given radius, perturbers at any angle $\phi$ produce perturbations stronger than $\Delta$. The limit $\delta(\theta, \phi)=\Delta$ is never achieved, not because perturbations are not strong enough, but because they are always too strong. In such cases, the desired angular interval is $\phi \in[0,2 \pi]$, so we define $\phi_{\mathrm{m}}=0$ for $\Delta>0$, and $\phi_{\mathrm{m}}=\pi / 2$ for $\Delta<0$.

We can now compute the magnification perturbation cross section by direct integration according to Eq. (3). This yields

$$
\sigma(\Delta)= \begin{cases}\pi \theta_{\max }^{2}-2 \int_{\theta_{\operatorname{man}}}^{\theta_{\max }}(\theta d \theta) 2 \phi_{m}(\theta \mid \Delta) & \text { if } \Delta>0 \\ 2 \int_{\theta_{\min }}^{\theta_{\max }}(\theta d \theta) 2 \phi_{m}(\theta \mid \Delta) & \text { if } \Delta<0 .\end{cases}
$$

Equation (6) is the result we were seeking. We summarize our algorithm for computing cross sections as follows.

1. First, given the convergence and shear profiles of a perturber, use Eq. (4) to find the maximum and minimum radii $\left(\theta_{\min }\right.$ and $\left.\theta_{\max }\right)$ such that perturbations $\delta$ stronger than $\Delta$ exist.

2. Second, define the function $\phi_{\mathrm{m}}(\theta \mid \Delta)$ via equation (5).

3. Third, use Eq. (6) to compute the total magnification perturbation cross section.

In appendices $\mathrm{B}$ and $\mathrm{C}$ we illustrate this formalism using point mass and singular isothermal sphere profiles as simple examples. This analysis reproduces the previous results of Mao \& Schneider (1998) and Keeton (2003) in these two cases respectively.

\section{THE EFFECTS OF MULTIPLE PERTURBERS: THE AVERAGE PERTURBATION AND ITS VARIANCE}

In the previous section, we studied the effect of a single perturber on an image and computed the cross sections for perturbations of a given amplitude. In this section, we extend these results and consider magnification perturbations due to an ensemble of perturbers. In particular, we compute the average perturbation $\left\langle\delta_{\mathrm{T}}\right\rangle$ and its variance in the limit that large magnification perturbations are unlikely and assuming that no individual perturber creates a perturbation larger than some linear cutoff $\Delta_{\mathrm{NL}}$.

Consider the magnification perturbation due to an ensemble of perturbers. Rather than adding these perturbers artificially and thereby increasing the mass of the lens, we assume the perturbers constitute a redistribution of some fraction of the mass of the macrolens. This is equivalent to adding substructure while at the same time adding a negative mass component that traces the macrolens' mass distribution. Thus, we first treat the problem of simply adding substructure artificially, and at the end we include the effect of mass conservation by adding the magnification perturbation due to a hypothetical negative mass component.

In our linear approximation, the total substructure magnification perturbation is the sum of the individual perturbations

$$
\delta_{\mathrm{T}}(N)=2|\mu|\left[(1-\kappa) \sum_{i=1}^{N} \delta \kappa_{i}-\gamma \sum_{i=1}^{N} \delta \gamma_{i} \cos \left(2 \phi_{i}\right)\right]=\sum_{i=1}^{N} \delta_{i}
$$

In Eq. (7), $\delta_{i}, \delta \kappa_{i}$, and $\delta \gamma_{i}$ represent the magnification perturbation, convergence perturbation, and shear perturbation of the $i$ th perturber respectively. This relation should hold provided that the position perturbation $\delta \boldsymbol{\alpha}$ is negligible. Unlike the case of a single perturber, in the case of multiple perturbers, we do not have a "rule-of-thumb" condition for when this approximation holds. However, we naïvely expect that the approximation will be good when magnification perturbations are small. In particular, oppositely-positioned perturbers give no net astrometric perturbation, but no analogous symmetry exists for magnification perturbations so long as $\delta \kappa>0$ (that is, the perturber represents an increase in the surface density). Therefore, we expect position perturbations to grow no faster than magnification perturbations. Henceforth, we assume Eq. (7) is valid (astrometric perturbations are negligible), and expect such an approximation to be justified if the net magnification perturbation is small, as in Eq. (1).

Given a perturber with a uniform probability of being in some finite region of space $\mathcal{R}$, about an image, the probability distribution $\rho(\delta)$, for a magnification perturbation in the range $\delta$ to $\delta+d \delta$ is given by

$$
\rho(\delta) d \delta=\frac{1}{A(\mathcal{R})} \int_{\mathcal{R}} d^{2} \boldsymbol{\theta} \delta_{\mathrm{D}}[\delta(\theta, \phi)-\delta]=\frac{1}{A(\mathcal{R})}\left|\frac{d \sigma}{d \delta}\right| d \delta,
$$

where $\sigma(\delta)$ is the cross section for the perturber to produce a perturbation stronger than $\delta, A(\mathcal{R})$ is the area of the region $\mathcal{R}$, and $\delta_{\mathrm{D}}[x]$ is the Dirac delta function of $x$. Though a uniform probability distribution for the perturber is unphysical, it is a reasonable approximation provided that the region over which perturbers can sizeably affect image magnifications is small compared to the length scale over which the projected number density of substructures varies.

We compute the various moments of the distribution $\left\langle\delta^{n}\right\rangle$, by defining the region $\mathcal{R}$ as all points $(\theta, \phi)$ such that $\delta_{\min }<$ $|\delta(\theta, \phi)|<\Delta_{\mathrm{NL}}$, In other words, we assume that the perturber creates a perturbation stronger than some minimum perturbation $\delta_{\min }$, but weaker than the cut off $\Delta_{\mathrm{NL}}$. Taking expectation values, and using the fact that $d \sigma(\delta) / d \delta<0$ for $\delta>0$ and $d \sigma(\delta) / d \delta>0$ for $\delta<0$ to integrate by parts, we obtain

$$
\begin{aligned}
\left\langle\delta^{n}\right\rangle & =\frac{1}{A(\mathcal{R})}\left[-\Delta_{\mathrm{NL}}^{n} y_{n}\left(\Delta_{\mathrm{NL}}\right)+\delta_{\min }^{n} y_{n}\left(\delta_{\min }\right)\right. \\
& \left.+n \int_{\delta_{\min }}^{\Delta_{\mathrm{NL}}} d \delta \delta^{n-1} y_{n}(\delta)\right],
\end{aligned}
$$

where we have defined the function $y_{n}(\delta)=\sigma(\delta)+(-1)^{n} \sigma(-\delta)$.

Consider an ensemble of identical perturbers characterized by a parameter vector $\mathbf{p}$ that specifies their projected mass density profiles (e.g., mass for point masses, or velocity dispersion for SIS perturbers). The expectation value of the total perturbation is

$$
\begin{aligned}
\left\langle\delta_{\mathrm{T}}\right\rangle & =\sum_{N=0}^{\infty} P(N) \int\left(\prod_{i=1}^{N} \rho\left(\delta_{i}\right) d \delta_{i}\right) \sum_{i=1}^{N} \delta_{i}(\mathbf{p}) \\
& =\langle N\rangle\langle\delta \mid \mathbf{p}\rangle,
\end{aligned}
$$

where the quantity $\langle\delta \mid \mathbf{p}\rangle$ is the expectation value of $\delta$ due to an individual perturber with parameters $\mathbf{p}$, and $P(N)$ is the probability of finding $N$ perturbers in the region $\delta_{\min } \leq|\delta| \leq \Delta_{\mathrm{NL}}$. A similar computation shows that the variance $\operatorname{VAR}\left(\delta_{\mathrm{T}}\right)=\left\langle\delta_{\mathrm{T}}^{2}\right\rangle-$ $\left\langle\delta_{\mathrm{T}}\right\rangle^{2}$ is given by

$$
\begin{aligned}
\operatorname{VAR}\left(\delta_{\mathrm{T}}\right) & =\langle N\rangle\left\langle\delta^{2} \mid \mathbf{p}\right\rangle+\left[\left\langle N^{2}\right\rangle-\langle N\rangle^{2}-\langle N\rangle\right]\langle\delta \mid \mathbf{p}\rangle^{2} \\
& =\langle N\rangle\left\langle\delta^{2} \mid \mathbf{p}\right\rangle,
\end{aligned}
$$


where, similar to the previous equation, $\left\langle\delta^{2} \mid \mathbf{p}\right\rangle$ is the expectation value of $\delta^{2}$ due to each individual perturber. To derive the second equation, we assumed that $P(N)$ is Poisson, such that $\operatorname{VAR}(N)=\langle N\rangle$, with no correlation in the spatial distribution of perturbers. ${ }^{9}$

We can extend this discussion to an ensemble of perturbers with different properties. Let $d s / d \mathbf{p}$ be the number density of perturbers with parameters $\mathbf{p}$ within some infinitesimal volume of parameter space $d \mathbf{p}$. The expected number of perturbers becomes $\langle N\rangle=D_{\mathrm{L}}^{2} A(\mathcal{R}) d s(\mathbf{p}) / d \mathbf{p}$, where $D_{\mathrm{L}}$ is the angular diameter distance to the lens. Placing this into our expressions for the average perturbation and summing over all perturbers (all parameters $\mathbf{p}$ ), we obtain

$$
\begin{aligned}
\left\langle\delta_{\mathrm{T}}\right\rangle & =\int d \mathbf{p} \frac{d s}{d \mathbf{p}} D_{\mathrm{L}}^{2}\left[-\Delta_{\mathrm{NL}} y_{1}\left(\Delta_{\mathrm{NL}}\right)+\delta_{\min } y_{1}\left(\delta_{\min }\right)\right. \\
& \left.+\int_{\delta_{\min }}^{\Delta_{\mathrm{NL}}} d \delta y_{1}(\delta)\right],
\end{aligned}
$$

where $\Delta_{\mathrm{NL}}$ and $\delta_{\min }$ are the maximum and minimum individual perturbations respectively. The total perturbation is obtained by taking the limit $\delta_{\min } \rightarrow 0$. Note that it is possible for the integral over $\delta$ to diverge (see SIS perturbers in appendix C). Likewise, the variance of the total perturbation is

$$
\begin{aligned}
\operatorname{VAR}\left(\delta_{\mathrm{T}}\right) & =\int d \mathbf{p} \frac{d s}{d \mathbf{p}} D_{\mathrm{L}}^{2}\left[-\Delta_{\mathrm{NL}}^{2} y_{2}\left(\Delta_{\mathrm{NL}}\right)+\delta_{\min }^{2} y_{2}\left(\delta_{\min }\right)\right. \\
& \left.+2 \int_{\delta_{\min }}^{\Delta_{\mathrm{NL}}} d \delta \delta y_{2}(\delta)\right] .
\end{aligned}
$$

Including mass conservation (the negative mass component mentioned above) is now trivial. If the mass density in substructures is $f_{\text {sub }} \Sigma_{\mathrm{c}} / 2$, the net perturbation becomes

$$
\left\langle\delta_{\mathrm{T}}\right\rangle=\left\langle\delta_{\mathrm{T}}\right\rangle_{\text {pert }}-|\mu|(1-\kappa) f_{\text {sub }}
$$

where the perturber contribution $\left\langle\delta_{\mathrm{T}}\right\rangle_{\text {pert }}$ is given by Equation (13) above and we used the fact that the convergence perturbation from the negative mass component is $\delta \kappa=-f_{\text {sub }} / 2$. This represents a constant contribution, so the variance remains unchanged. In Appendices B and $\mathrm{C}$, we consider the average perturbation $\left\langle\delta_{\mathrm{T}}\right\rangle$ and its variance $\operatorname{VAR}\left(\delta_{\mathrm{T}}\right)$ for the case of point mass and SIS perturbers respectively.

\section{MAGNIFICATION PERTURBATIONS BY HALO SUBSTRUCTURES}

In this section we apply our formalism to the problem of magnification perturbations by substructures within the context of a simple, but realistic, model of CDM substructure that includes a mass spectrum of perturbers and tidal truncation of the substructure profiles. We find that such perturbations are local in that the assumption of a constant surface density of perturbers is valid. We find that while the most massive perturbers dominate the total magnification perturbation, the latter has significant contributions from a large range of substructure masses and these contributions depend upon the shape of the substructure mass spectrum. In what follows, we ignore the negative mass component perturbation in $\S 4.1, \S 4.2$, and $\S 4.3$, which deal with the details of computing the granular contribution to the magnification perturbation. We include the negative mass contribution in $\S 4.4$, where we illustrate the expectation values for the specific models.

${ }^{9}$ Strictly speaking, one would expect the perturbers to be correlated. However, if the surface density of perturbers is sufficiently low, such correlations should be a small effect.

\subsection{Substructure Perturbations Are Local}

Following the analysis of Dalal \& Kochanek (2002a), we adopt a simplified model for substructures in which perturbers are characterized by so-called pseudo-Jaffe mass density profiles (see Muñoz et al. 2001). The three-dimensional density distribution is

$$
\rho(r) \propto \frac{1}{r^{2}\left(r^{2}+a^{2}\right)},
$$

where the parameter $a$ is an effective tidal radius that should physically be present for all substructures and serves to localize the total mass of the perturber. ${ }^{10}$ The two-dimensional convergence and shear profiles for the pseudo-Jaffe model are given by

$$
\delta \kappa=\tilde{b}\left(\frac{1}{2 \theta}-\frac{1}{2 \xi}\right)
$$

and

$$
\delta \gamma=\frac{\tilde{b}}{2 \theta}+\frac{\tilde{b}}{2 \xi}-\frac{\tilde{b} a}{\theta^{2}}\left(\frac{\xi}{a}-1\right)
$$

where $\tilde{b}$ is defined by the combination $b / \tilde{b}=1+a / b-(1+$ $\left.(a / b)^{2}\right)^{1 / 2}$, the quantity $b$ is the Einstein radius of the profile, and $\xi \equiv \sqrt{\theta^{2}+a^{2}}$. When $a \rightarrow \infty$, the profiles approach that of an SIS perturber with an Einstein radius $b=\tilde{b}$, while in the limit $a \rightarrow 0$ with $a \tilde{b}$ constant, the perturber corresponds to a point mass with Einstein radius $b=\sqrt{a \tilde{b}}$. The total mass of the perturber is given by $m=\pi a \tilde{b} \Sigma_{\mathrm{c}} D_{\mathrm{L}}^{2}$.

First, consider the average perturbation $\left\langle\delta_{\mathrm{T}}\right\rangle$, for a positiveparity image. Perturbers well within a tidal radius from the image act as SIS $(\delta \kappa=\delta \gamma \sim 1 / \theta)$, so each individual perturbation $\delta$ scales as $\delta \sim \delta \kappa \sim 1 / \theta$, while the number of perturbers per logarithmic interval grows as $\sim \theta^{2}$. As a result, the contribution to $\delta_{\mathrm{T}}$ scales as $\sim \theta \sim 1 / \delta$, meaning that it increases with decreasing individual perturber strength, until a peak at $\delta_{\mathrm{p}} \approx|\mu|(1-\kappa) b / a$, which is the typical perturbation from substructure one tidal radius away from the image.

At large distances the behavior is slightly more complicated. One might naïvely expect point mass behavior to dominate, but the average perturbation due to point masses is zero. A nonzero contribution to $\delta_{\mathrm{T}}$ arises from the small, but nonzero, convergence $\delta \kappa \sim 1 / \theta^{3}$ (valid for $\theta \gg a$ ). Therefore, the contribution from perturbers a distance $\theta$ away scales as $\sim \theta^{2} \delta \kappa \sim 1 / \theta$, which, for convergence-dominated perturbations, is a $\sim \delta^{1 / 3}$ scaling. In the limit $\delta \rightarrow 0$, all perturbations eventually become shear dominated with the individual perturber strength $\delta \sim 1 / \theta^{2}$, so the net contribution to $\delta_{\mathrm{T}}$ scales as $\sim 1 / \theta \sim \delta^{1 / 2}$.

We illustrate this behavior explicitly in Figure 2, where we assume typical macromodel parameters $\kappa=0.5$ and $\gamma=0.2$. For comparison, we also present the case for truncated SIS perturbers. At first glance, the curve for truncated SIS perturbers seems puzzling because the curve does not drop off to zero at the tidal radii $\left(\delta<\delta_{\mathrm{p}}\right)$. The reason for this behavior is that we have been somewhat loose in our interpretation of $y_{1}(\delta) \delta$. In

\footnotetext{
${ }^{10}$ We note that while a pseudo-Jaffe profile is not the most accurate treatment for the density profiles of dark matter substructures observed in simulations, it is qualitatively correct in that the matter density quickly drops beyond a truncation radius $a$. Due to its simplicity, and because it allows us to better relate to previous work which uses this or related profiles, we have opted to model substructures as pseudo-Jaffe profiles. Adiabatic contraction is one motivation for using steeper inner profiles, but this is likely to be irrelevant for the majority of small, subhalos that do not host luminous galaxies. The formalism that we present can be adapted to accommodate more general subhalo density profiles.
} 


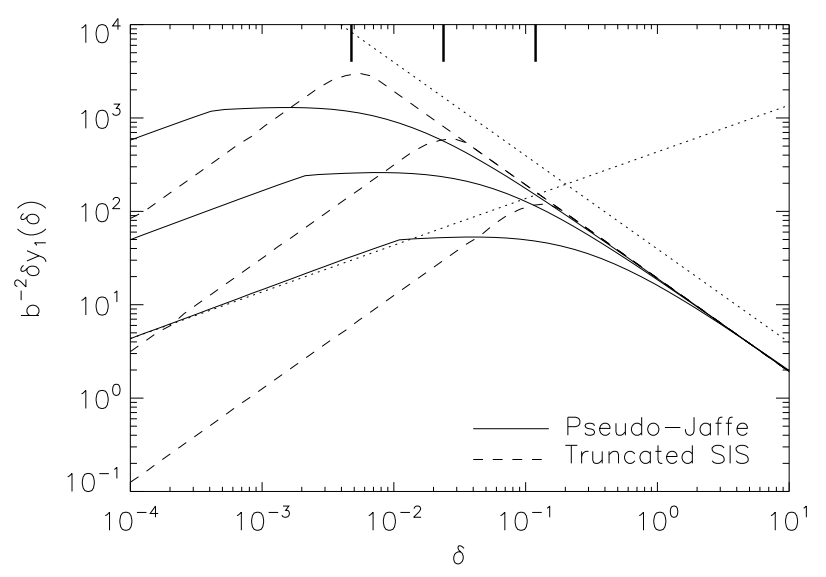

FIG. 2.- The contribution per logarithmic interval to the total perturbation due to perturbers of strength $\delta$. The quantity $\delta y_{1}(\delta)$ (see text) must be multiplied by a number density to get a perturbation, so we plot the dimensionless quantity $b^{-2} \delta y_{1}(\delta)$ where $b$ is the perturber's Einstein radius. All of the curves assume a macromodel with $\kappa=0.5$ and $\gamma=0.2$. From top to bottom the three solid and dashed lines correspond to perturbers with $a / b=500,100,20$, where $a$ is the substructure tidal radius. The solid lines show the relation for pseudo-Jaffe profiles [cf. Eq. (16), Eq. (17), Eq. (18)]. The dashed lines are truncated SIS profiles. The falling dotted line shows the expected scaling for SIS perturbers, $\delta y_{1}(\delta) \propto 1 / \delta$, while the rising dotted line shows the expected scaling in the limit $\delta \rightarrow 0$. The broad peak for the pseudo-Jaffe profiles reflects the smooth change from $\delta y_{1} \propto \delta^{-1}$ to $\propto \delta^{1 / 3}$. This transitions to $\propto \delta^{1 / 2}$ when perturbations become shear dominated (sharp break). The peak scales $\delta_{\mathrm{p}}=|\mu|(1-\kappa) b / a$ are marked with short, vertical lines at the top of the Figure.

particular, the full contribution to $\delta_{\mathrm{T}}$ per logarithmic interval is $\delta \rho(\delta)=\delta d y_{1} / d \delta$, which is zero in the region $\delta<\delta_{\mathrm{p}}$. To derive Eq. (13), we integrated $\delta d y_{1}(\delta) / d \delta$ by parts. This halves the contribution from the region $\delta>\delta_{\mathrm{p}}$, while introducing a symmetrical contribution in the region to the left of the peak.

Analogous arguments hold for the variance of the magnification perturbations, $\operatorname{VAR}\left(\delta_{\mathrm{T}}\right)$. Perturbers within a tidal radius contribute $\sim \theta^{2} d \ln (\theta) \delta^{2} \sim d \ln (\delta)$ to the variance, so all perturbers of strength $\delta<\delta_{\mathrm{p}}$ contribute at comparable levels to $\operatorname{VAR}\left(\delta_{\mathrm{T}}\right)$. Perturbers far away act as point masses and contribute an amount that scales as $\sim \theta^{2} \delta^{2} \sim \delta$, where we used $\delta \sim 1 / \theta^{2}$ for point masses. Finally, there is a small transition region between the peak and point-mass behavior where perturbations are convergence dominated with $\delta \kappa \sim 1 / \theta^{3}$, corresponding to $\mathrm{a} \sim \delta^{4 / 3}$ scaling. We show the contribution to the variance from strength $\delta$ perturbers in Figure (3).

The above results have some interesting implications. In particular, we have demonstrated that: (1) the average perturbation is dominated by perturbers that are about one tidal radius away from the image; and (2) the variance of the perturbations weighs all perturbers within a tidal radius equally, with perturbers further away contributing relatively little. In other words, magnification perturbations are effectively "local" to within the typical tidal radii of the perturbers. How local is this? Consider a perturber a distance $d$, from the center of the macrolens potential. Then $d=\sqrt{r^{2}+z^{2}}$, where $r$ is its projected distance onto the lens plane and $z$ is the separation along the line of sight. Assuming, for simplicity, truncated SIS profiles for both host and perturber, we can approximate the tidal radius $a(r, z)$, by setting $\rho_{\text {perturber }}(a)=\rho_{\text {host }}(d)$. We are generally interested in the properties of substructures at a projected distance roughly equal to the Einstein radius of the macrolens, $r=b_{H}$. We find

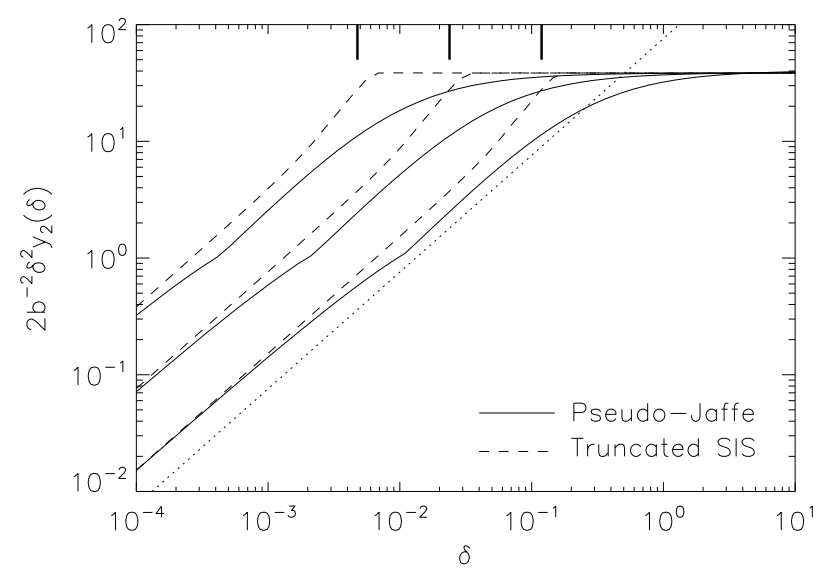

FIG. 3.- The contributions per logarithmic interval to the variance of magnification perturbations. The lines are the same as in Figure 2, but the vertical axis now represents the contribution to the variance due to perturbers of strength $\delta$ in the dimensionless form $b^{-2} \delta^{2} y_{2}(\delta)$. Again, the macromodel has $\kappa=0.5$ and $\gamma=0.2$, and the substructure Einstein $(b)$ and tidal $(a)$ radii satisfy $a / b=500,100,20$ from left to right. The dotted line is the expected scaling for point masses, $\delta^{2} y_{2}(\delta) \propto \delta$, and the short, vertical lines at the top mark the peak scale $\delta_{\mathrm{p}}=|\mu|(1-\kappa) b / a$.

$(b / a)^{2}=b b_{H} /\left(b_{H}^{2}+z^{2}\right)$. Assuming, for simplicity, that substructures trace the smooth component, we can obtain an estimate for the average value of $b / a$ along the line of sight to the lens by integrating along the line-of-sight distance, $z$. This yields

$$
\langle b / a\rangle \approx \frac{1}{\lambda_{a}}(2 / \pi)\left(b / b_{H}\right)^{1 / 2},
$$

with $\lambda_{a}=1$. For comparison, Dalal \& Kochanek (2002a) used $\lambda_{a}=2 / \pi \simeq 0.64$. We introduced the parameter $\lambda_{a}$, as a way to change the effective tidal radii in our model. Larger $a$ corresponds to systematically less centrally-concentrated density profiles. Kochanek \& Dalal (2004) assumed $\lambda_{a}=2 / \pi$ in their analysis. For $b / b_{H} \sim 10^{-3}-10^{-2}$ (e.g., milliarcsecond perturbers), we obtain $a / b_{H} \approx 5 \%-15 \%$. This distance is small enough that perturbations may be considered local in the sense that the two-dimensional surface density of perturbers does not vary much on this length scale (cf. Zentner \& Bullock 2003; Mao et al. 2004; Diemand et al. 2004; Nagai \& Kravtsov 2005), but large enough that magnification perturbations to nearby pairs of images may be correlated, especially if the substructure profiles are less centrally concentrated than we have assumed $\left(\lambda_{a}>1\right)$.

\subsection{Dependence of Magnification Perturbations on the Substructure Mass Spectrum}

We now investigate the dependence of magnification perturbations on the mass spectrum of the substructure perturbers. Consider Equation (13) for the average perturbation. We approximate the mass spectrum as a power law $d s / d m \propto m^{\alpha}$, truncated at some maximum mass $m_{\max }$, where $d s / d m$ is the number density of substructures in the mass range from $m$ to $m+d m$. The slope $\alpha$ has been determined by high-resolution, cosmological $N$-body simulations, which generally yield $\alpha \approx-1.8 \pm 0.1$ (e.g., Klypin et al. 1999; Moore et al. 1999; De Lucia et al. 2004; Diemand et al. 2004; Gao et al. 2004). The surface mass fraction $d f_{\text {sub }}$ of perturbers of mass $m$ is $(1 / 2) \Sigma_{\mathrm{c}} d f_{\text {sub }}=d \Sigma_{\mathrm{s}}=$ $m d s$, so that the mass fraction varies as $d f_{\text {sub }} / d m \propto m^{\alpha+1}$. Here, we have chosen to follow Dalal \& Kochanek (2002a) and define 


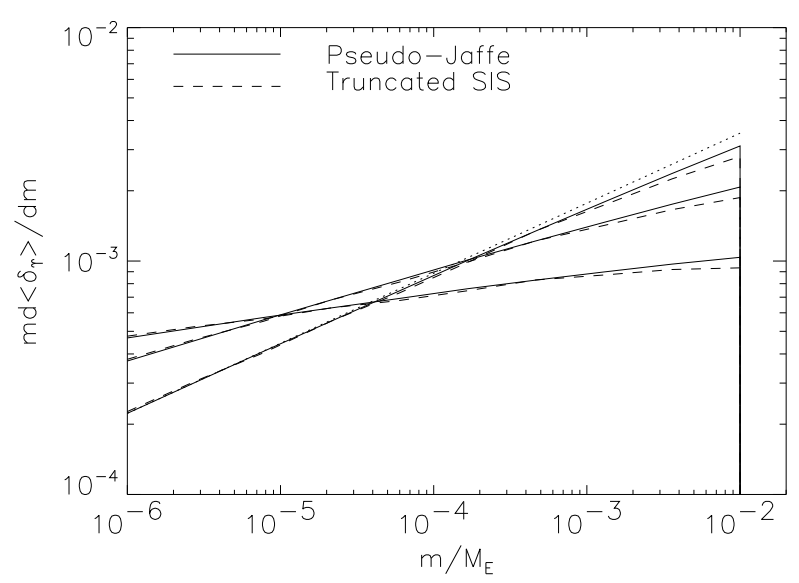

FIG. 4.- The average magnification perturbation per logarithmic interval in substructure mass $m$. Masses are expressed in units of the smooth lens mass $M_{E}$ contained within the Einstein ring, and the most massive substructures considered are $m_{\max } / M_{E}=1 \%$ (see text). A substructure mass fraction of $f_{\text {sub }}=5 \times 10^{-3}$ with a power-law spectrum of index $\alpha$ was assumed. The macromodel parameters are $\kappa=0.5$ and $\gamma=0.2$. The solid lines are for the pseudo-Jaffe profiles, and the dashed are for truncated SIS profiles. From top to bottom at right, the value of $\alpha$ is $\alpha=-1.7,-1.8$, and -1.9 . The dotted line shows the expected $m^{2+\alpha}$ scaling for $\alpha=-1.7$.

the "mass fraction" $f_{\text {sub }}$ relative to half of the critical density, $f_{\text {sub }}=2 \Sigma_{\mathrm{s}} / \Sigma_{\mathrm{c}}$. This choice is motivated by the fact that all images in a lens will probe similar radii in the host halo, and thus similar substructure densities, whereas the macromodel convergence may vary more strongly from image to image. Note that the quantity $m d s / d m$ is precisely the number of perturbers of mass $m$ per logarithmic mass interval, so that Eq. (13) can be recast as

$$
\begin{aligned}
\left\langle\delta_{\mathrm{T}}\right\rangle & \approx \frac{1}{2} \int_{0}^{m_{\max }} \frac{d m}{m} \frac{d f_{\mathrm{sub}}}{d m} \Sigma_{\mathrm{c}} D_{\mathrm{L}}^{2} \int_{\delta_{\min }}^{\Delta_{\mathrm{NL}}} d \delta y_{1}(\delta) \\
& \approx \frac{1}{2}(\alpha+2) f_{\text {sub }} \int_{0}^{m_{\max }} \frac{d m}{m}\left(\frac{m}{m_{\max }}\right)^{\alpha+2} \\
& \times\left[\frac{\Sigma_{\mathrm{c}} D_{\mathrm{L}}^{2}}{m} \int_{\delta_{\min }}^{\Delta_{\mathrm{NL}}} d \delta y_{1}(\delta)\right],
\end{aligned}
$$

where we have ignored the contribution from the boundary terms by taking the limits $\delta_{\min } \rightarrow 0$ and $\Delta_{\mathrm{NL}} \rightarrow \infty$, and we have normalized the substructure fraction as $f_{\text {sub }}=$ $\int_{0}^{m_{\max }} d m d f_{\text {sub }} / d m$, neglecting a possible low-mass cutoff by assuming that $\alpha>-2$. The mass $m_{\max }$ represents the mass scale of the most massive substructures contributing to the magnification perturbations. Below, we argue that this is given roughly by $m_{\max } / M_{E} \sim 1 \%$, where $M_{E}$ is the mass of the macrolens contained within an Einstein radius in projection.

With these assumptions we can estimate how the integrand in Eq. (20) scales with mass. The perturbation by mass $m$ perturbers is dominated by substructures a tidal radius away from the image and scales as $\sim a^{2}(|\mu| b / a) \sim a b \sim m$. Therefore, the term in square brackets is roughly constant, and the perturbation per logarithmic mass interval varies as $\sim m^{2} d s \sim m d f_{\text {sub }} \sim$ $m^{\alpha+2}$ or as $\sim m^{0.1-0.3}$ for $\alpha=-1.7$ to -1.9 . This dependence is rather weak. We show the $m^{2+\alpha}$ scaling explicitly in Figure 4, after enforcing a linear cutoff $\Delta_{\mathrm{NL}}=1$ (replacing the boundary term that we neglected earlier). We show curves for both pseudo-Jaffe and truncated SIS profiles. As is evident in the
Fig. 4, the results are nearly identical for these two cases. Finally, the term in the square brackets in Equation (20) is, to a good approximation, a constant function of perturber mass, so that we can write

$$
\left\langle\delta_{\mathrm{T}}\right\rangle \approx \frac{1}{2} f_{\text {sub }}\left[\frac{\Sigma_{\mathrm{c}} D_{\mathrm{L}}^{2}}{m} \int_{\delta_{\min }}^{\Delta_{\mathrm{NL}}} d \delta y_{1}(\delta)\right]_{m=m_{\max }} .
$$

The error introduced by making this approximation is $\approx 5 \%$ and is nearly independent of the exact value of $m_{\max }$ so long as the peak scale $\delta_{\mathrm{p}}<\Delta_{\mathrm{NL}}$, where we reiterate that $\Delta_{\mathrm{NL}}$ is a linear cutoff scale.

As before, we can construct a similar argument for the variance of the magnification perturbations. In this case, all perturbers within a tidal radius contribute equally to the variance, so a linear cutoff $\Delta_{\mathrm{NL}}$, needs to be introduced. This constant contribution scales as $\sim a^{2}(b / a)^{2} \sim b^{2} \sim m^{4 / 3}$, and is integrated between the peak scale $\delta_{\mathrm{p}}$ and the linear cutoff $\Delta_{\mathrm{NL}}$. The contribution per logarithmic mass interval is thus $\sim\left(m d f_{\text {sub }}\right) m^{4 / 3} \ln \left(\Delta / \delta_{\mathrm{p}}\right) \sim m^{\alpha+7 / 3} \ln \left(\Delta / \delta_{\mathrm{p}}\right)$. For $\alpha=-1.8$, this is $\sim m^{8 / 15} \ln \left(\Delta / \delta_{\mathrm{p}}\right)$. As before, we can use our understanding of this behavior to provide a simple estimate of the variance,

$$
\begin{aligned}
\operatorname{VAR}\left(\delta_{\mathrm{T}}\right) & \approx \frac{1}{2}(\alpha+2) f_{\text {sub }}\left[\frac{2 \Sigma_{\mathrm{c}} D_{\mathrm{L}}^{2}}{m} \int_{0}^{\Delta_{\mathrm{NL}}} d \delta \delta y_{2}(\delta)\right]_{m=m_{c}} \\
& \times \int_{0}^{m_{\max }} \frac{d m}{m}\left(\frac{m}{m_{\max }}\right)^{2+\alpha+\frac{1}{3}}\left[\frac{\ln \left(\Delta / \delta_{\mathrm{p}}(m)\right)}{\ln \left(\Delta / \delta_{\mathrm{p}}\left(m_{\max }\right)\right)}\right](22)
\end{aligned}
$$

This approximation is valid to about $\approx 5 \%$ and, once again, it breaks down as $\delta_{\mathrm{p}}$ approaches $\Delta_{\mathrm{NL}}=1$. We illustrate these results in Figure 5, and we note particularly the flatness of the variance per logarithmic mass interval, which is shallower than $d \ln [\operatorname{VAR}(d t)] / d \ln m \lesssim 1 / 2$. Note that the expression above differs from that of identical mass $m_{\max }$ perturbers, demonstrating that the statistical properties of magnification perturbations by substructures depend on the mass spectrum of the perturbers. This is not surprising as such a dependence has been noted in the case of microlensing (e.g., Schechter et al. 2004). Finally, from Figure 5, we see that SIS and pseudo-Jaffe profiles lead to different variances at a fixed mass fraction, implying that the variance of the magnification perturbation is sensitive to the substructure mass density profile.

\subsection{Truncating the Substructure Mass Spectrum}

It is interesting to consider what the appropriate values for the effective minimum and maximum masses, $m_{\max }$ and $m_{\min }$, are. We begin by considering the relatively simpler problem of the low-mass cutoff, $m_{\min }$. In general, the magnification perturbation due to a perturber smaller than the physical extent of the source region is small (e.g., Schneider et al. 1992; Dobler \& Keeton 2005). The average perturbation is dominated by perturbers a distance $\simeq a$ from the source, where $a$ is the tidal radius of the substructure, so the point source approximation should be valid so long as the characteristic size of the source $R$ is smaller than $a$. The minimum substructure mass can therefore be obtained by setting $a \approx R$. Taking $R \sim 10 \mathrm{pc}$, as appropriate for quasar radio-emission regions, we find $m_{\min } / M_{E} \gtrsim \lambda_{a}^{-2} 10^{-9}$, small enough to neglect for our present purposes ${ }^{11}$.

11 One could require instead $b \approx R$, which is appropriate in the limit that individual perturbations dominate the net magnification perturbation. This gives 


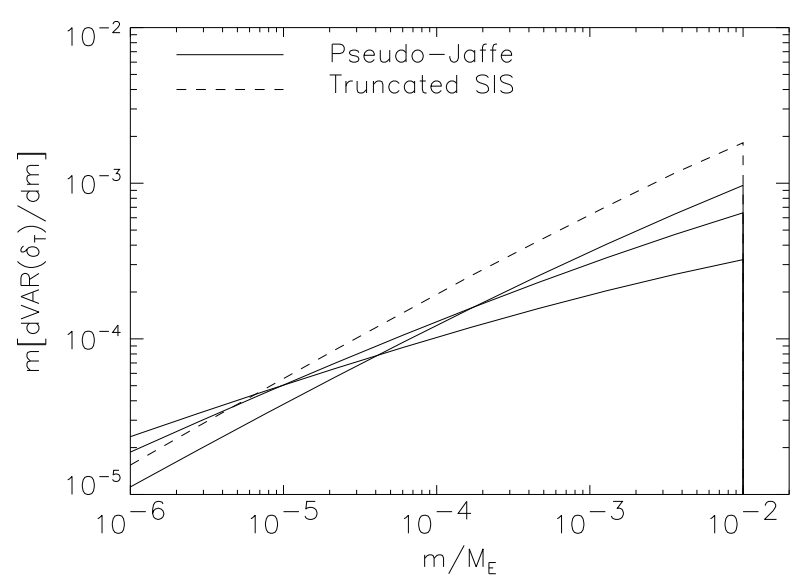

FIG. 5.- The variance of the magnification perturbation per logarithmic mass interval. A substructure mass fraction of $f_{\text {sub }}=0.5 \%$ with a power-law spectrum of slope $\alpha$ was assumed. The linear cutoff $\Delta_{\mathrm{NL}}$ is set to unity and the macromodel parameters are $\kappa=0.5$ and $\gamma=0.2$. The solid lines are for the pseudo-Jaffe profile and the value of $\alpha$ is, from top to bottom at right, $\alpha=-1.7,-1.8$, and -1.9 . The dashed line is for a truncated SIS profile with $\alpha=-1.7$.

Estimating the appropriate maximum effective substructure mass $m_{\max }$, is a considerably more difficult problem. In the context of CDM, the most massive substructures within a halo have masses that are typically of order $m_{\max } \sim 10^{-2} M_{\mathrm{vir}}$, where $M_{\mathrm{vir}}$ is the virial mass of the halo (e.g., Klypin et al. 1999; Moore et al. 1999; Ghigna et al. 2000; De Lucia et al. 2004; Diemand et al. 2004; Gao et al. 2004). For a halo with $M_{\text {vir }}=10^{13} M_{\odot}$, this corresponds to $m \sim 10^{11} M_{\odot}$, which is comparable to the mass of the host halo contained within a cylinder of radius equal to the Einstein radius of the lens. Clearly, if such a substructure were to fall near the Einstein radius of the host halo, all images would be strongly perturbed and the lens macromodel would be significantly altered (see, e.g., Cohn \& Kochanek 2004, and references therein). In the most naïve view, one could imagine that such effects are either entirely degenerate with the macromodel, or else explicitly including them when the substructures can be optically identified. In this naïve picture, magnification perturbations come about only through substructures that do not affect the macromodel. Taking the primary macromodel property to be the Einstein radius, one can demand that $m_{\max } \ll M_{E} \propto b_{H}^{2}$, so one may expect $m_{\max } / M_{E}=1 \%$ to be a reasonable cutoff mass.

A more pragmatic self-consistency argument can also be made. We have assumed in our formalism that we can neglect astrometric perturbations relative to the magnification perturbations, so we should include only perturbers for which the characteristic perturbation scale $\delta_{\mathrm{p}}$ is linear. We can then define $m_{\max }$ by setting $\delta_{\mathrm{p}}\left(m_{\max }\right)=1$, which leads to $m_{\max } \sim$ $\lambda_{a}^{4}(\pi / 2)^{4}|\mu|^{-3}$. For $\lambda_{a}=1,|\mu|=10$, and $\delta_{\mathrm{p}}=1$, we get $m_{\max } / M_{E} \sim 0.6 \%$, though it is clear that this value depends upon the various parameters. However, it is important to note that less centrally-concentrated profiles $\left(\lambda_{a}>1\right)$ allow for significantly larger mass cutoffs, implying that linear theory becomes a better approximation. In what follows, we truncate the

$m_{\min } / M_{E} \gtrsim \lambda_{a} 10^{-5}$. In our model, the number of nonlinear perturbers of mass $m$ scales as $m^{\alpha+1} b^{2} \sim m^{\alpha+7 / 3} \sim m^{1 / 2}$, implying that the number of nonlinear perturbers increases with mass. At most, or order one nonlinear perturbers will be present, and these will all be massive. Hence, the appropriate limit for $m_{\min }$ is that of linear perturbers obtained by setting $R \approx a$ as above.

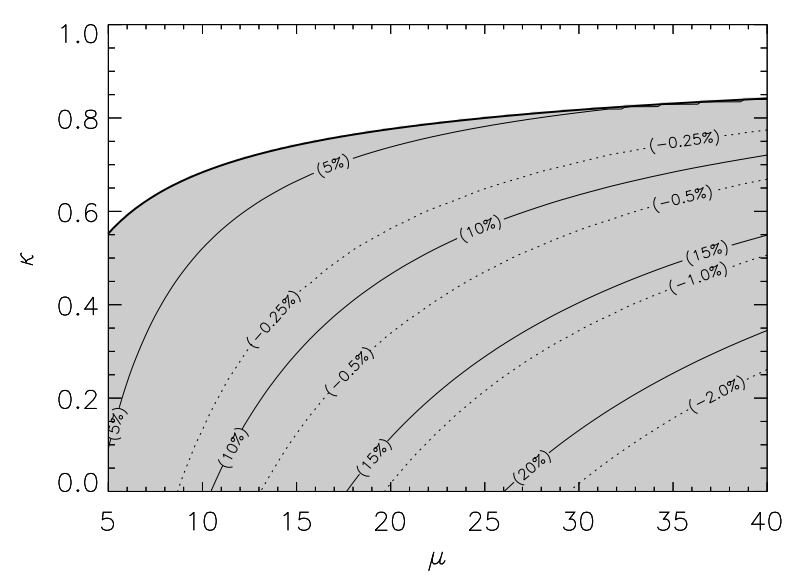

FIG. 6. - The average and rms values for the total magnification perturbation due to linear perturbers of an image with macromodel magnification $\mu$ and convergence $\kappa$. Solid contours give the rms values while dotted contours give the average perturbation. Lens and source redshifts of $z_{1}=0.3$ and $z_{\mathrm{s}}=1$ respectively, were assumed, and a high-mass cutoff $m_{\max } / M_{E}=1 \%$, where $M_{E}$ is the mass of the macrolens projected to within an Einstein radius. The tidal radii were set by $\lambda_{a}=4$. The substructure mass fraction is $f_{\text {sub }}=0.5 \%$, and mass conservation is enforced through a negative mass component of surface density $-\kappa_{s}=2 \Sigma_{\mathrm{c}} f_{\text {sub }}$. The unshaded region is unphysical as it corresponds to $\gamma<0$. The rms scatter is comparable to that shown in Figure 3 of Kochanek $\&$ Dalal (2004).

mass spectrum at $m_{\max } / M_{E}=1 \%$, regardless of the macromodel parameters.

It should be clear from these arguments that the truncation scale $m_{\max }$ carries with it a some uncertainty. In general, any given macromodel should be able to partly, but not fully, compensate for perturbations due to very massive substructures, in which case there will be an additional contribution to the observed magnification perturbations that is not being included by introducing a cutoff $m_{\max } / M_{E}=1 \%$. Estimating the degree to which the effect of massive substructures can be absorbed within the macromodel is beyond the scope of this work, but we explore this in a forthcoming companion paper (Chen \& Rozo 2005, In preparation).

\subsection{General Predictions}

We can use this formalism to make some general predictions regarding magnification perturbations due to halo substructure. Figure 6 illustrates predictions for the average and rms value of the total magnification perturbation in the case of positiveparity images. As an illustrative example, we have assumed a source redshift of $z_{\mathrm{s}}=1$ and a lens redshift of $z_{1}=0.3$. We have also assumed a high-mass cutoff $m_{\max } / M_{E}=1 \%$, a substructure mass fraction $f_{\text {sub }}=2 \Sigma_{s} / \Sigma_{\mathrm{c}}=0.5 \%$, a tidal radius given by $\lambda_{a}=4$, and a typical host Einstein radius of $b_{H}=1$ ". Although the average magnification perturbations are very small $(\lesssim 1 \%)$, the rms fluctuations are quite large $\left(\delta_{\mathrm{T}}^{\mathrm{rms}} \gtrsim 10 \%\right)$, and hence large magnification perturbations should be common. Our rms estimates seem comparable to the scatter in Kochanek \& Dalal $\left(2004, \delta_{\mathrm{T}}^{\mathrm{rms}} \approx 10-15 \%\right.$, compare to their Figure 3$)$, though the average perturbation seems markedly different, even at a qualitative level. Particularly, we find small $\left(\left|\left\langle\delta_{\mathrm{T}}\right\rangle\right| \ll \delta_{\mathrm{T}}^{\mathrm{rms}}\right)$, negative average perturbations, whereas Kochanek \& Dalal (2004) report large $\left(\left|\left\langle\delta_{\mathrm{T}}\right\rangle\right| \sim 10 \% \sim \delta_{\mathrm{T}}^{\text {rms }}\right)$, positive average perturbations.

How serious is the discrepancy between the model and ob- 
served lenses and what is it telling us? To address this question, we consider in some more detail our expression for the average perturbation. We now show that small negative perturbations are a generic prediction of linear perturbation theory when the total mass of the lensing potential is held fixed. Consider again our expression for the total magnification perturbation, Equation 7. For simplicity, assume that all perturbers are identical. Consider the shear contribution to the average perturbation. Two perturbers equidistant from an image and located at position angles $\phi$ and $\phi+\pi / 2$ create equal and opposite shears so the net shear contribution to the average perturbations is zero, to a first approximation. The only nonzero contribution is caused by the convergence perturbations, so we can write

$$
\left\langle\delta_{\mathrm{T}}\right\rangle \simeq 2|\mu|(1-\kappa)\langle\delta \kappa\rangle,
$$

where the expectation value of the convergence perturbation is yet to be determined. An argument similar to the one in $\S 3$ results in

$$
\langle\delta \kappa\rangle=\sum_{N} P(N) N \int_{\mathcal{R}} \frac{d^{2} \theta}{A(\mathcal{R})} \delta \kappa(\theta),
$$

where the region $A(\mathcal{R})$ is defined as before (i.e. $\theta \in \mathcal{R}$ iff $\left.\delta_{\min }<\delta(\boldsymbol{\theta})<\Delta_{\mathrm{NL}}\right)$ and $N$ is the number of perturbers. We assume that $P(N)$ is a Poisson distribution with an expectation value $\langle N\rangle=s A(\mathcal{R})$, where $s$ is the mean number density of perturbers. Observe that if the integral over $\kappa$ in the above expression were extended over all space, we would recover the mass of the perturber. This leads us to define the nonlinear and linear mass fractions as

$$
f_{\mathrm{NL}}=\frac{\sum_{\mathrm{c}} D_{\mathrm{L}}^{2}}{m} \int_{|\delta|<\Delta_{\mathrm{NL}}} d^{2} \theta \delta \kappa(\theta),
$$

and $f_{\mathrm{L}}=1-f_{\mathrm{NL}}$ respectively, where $m$ is the perturber mass. Thus, $f_{\mathrm{NL}}$ is the fraction of the perturber mass contained within the nonlinear region around an image for a perturber centered on the image. Using these definitions, and taking the limit $\delta_{\min } \rightarrow 0$, Eq. (24) becomes

$$
\langle\delta \kappa\rangle=\langle N\rangle \frac{m\left(1-f_{\mathrm{NL}}\right)}{\Sigma_{\mathrm{c}} D_{\mathrm{L}}^{2} A}=\kappa_{s}\left(1-f_{\mathrm{NL}}\right),
$$

where $\kappa_{s}=s m /\left(\Sigma_{\mathrm{c}} D_{\mathrm{L}}^{2}\right)$ is just the convergence contribution by substructures of mass $m$. Replacing this contribution in our expression for the average perturbation, and including the negative mass component $-\kappa_{s}$, we obtain

$$
\left\langle\delta_{\mathrm{T}}\right\rangle_{\mathrm{LIN}}=-2|\mu|(1-\kappa) \kappa_{s} f_{\mathrm{NL}} .
$$

Thus, the average perturbation due to linear perturbers is always expected to be negative and approaches zero in the limit that local, linear perturbation theory holds. Indeed, our choice $\lambda_{a}=$ 4 for Figure 6 was made to make $f_{N L}$ small so as to minimize nonlinear effects. Lowering $\lambda_{a}$ increases the nonlinear fraction $f_{N L}$ and hence $\left\langle\delta_{\mathrm{T}}\right\rangle$ becomes more negative $\left(f_{N L} \propto 1 / \sqrt{\lambda_{a}}\right.$ at fixed mass).

It is important to emphasize that a negative $\left\langle\delta_{\mathrm{T}}\right\rangle$ implies that positive-parity images will be dimmed and negative-parity images will be brightened, which is opposite from the trend for observed lenses (Kochanek \& Dalal 2004), provided one uses the predicted macromodel magnification as a proxy for the observed magnification if the substructures were replaced by a smooth component. Because we assumed only that perturbations are linear and local, the implications are that either real lenses are significantly affected by nonlinear perturbers, perturbations are at least partly due to nonlocal perturbers, or the

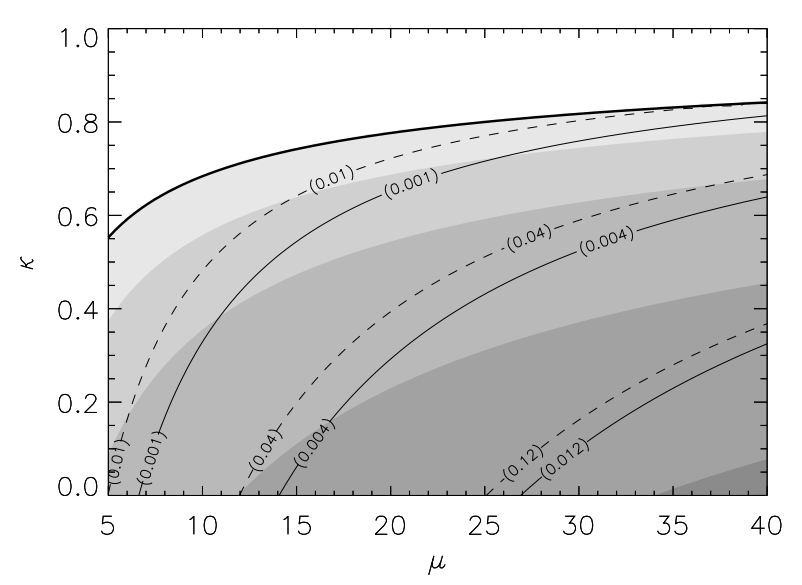

FIG. 7.- The expected number of substructures for various conditions. The unshaded region above the thick, solid line is unphysical for positive parity images. The shaded regions below are obtained by computing the expected number of perturbers that create perturbations stronger than $\delta_{\mathrm{p}}$ (see Figure 2), and correspond, from top to bottom, to $\left\langle N \mid \delta>\delta_{p}\right\rangle \in$ $[0,20],[20,26],[26,29],[29,30]$, and 30 or more perturbers. All substructures parameters are as per section 4.4. We loosely interpret $\left\langle N \mid \delta>\delta_{p}\right\rangle$ as the number of substructures found within a tidal radius of the image (see text). The solid contours are for $\langle N \mid \delta>1\rangle$, and give the expected number of nonlinear perturbers, which is seen to by quite small, $\lesssim 10^{-2}$. The dashed contours are for a more conservative linear cutoff $\Delta_{\mathrm{NL}}=20 \%$, for which we find $\langle N \mid \delta>20 \%\rangle \lesssim 0.1$. Truncated SIS profiles give results similar to that of the pseudo-Jaffe profiles.

smooth macromodel magnification is a poor proxy for what the magnification would be in the absence of substructures. Note that these various possibilities are not mutually exclusive. Of course, an alternative, and perhaps more contentious, possibility is simply that substructures within lens galaxies are not responsible for the observed magnification perturbations. Assuming this last possibility is incorrect, what is the most likely explanation for our results?

Nonlocal perturbations do not appear to be the most reasonable resolution. So long as linear perturbation theory holds, our naïve expectation is that the average convergence perturbations, and hence the average magnification perturbation itself, should be close to zero. Further, the two-dimensional surface number density of substructures predicted for CDM halos is a very slowly-varying function of radius out to several times the Einstein radius of the lens, so a constant perturber density seems like a reasonable approximation. Thus, we do not view nonlocal (non-constant substructure surface density) perturbations as a viable explanation for the observed discrepancy. However, it is worth noting that if nonlocal perturbations play a significant role, naïve comparisons of the substructure mass fractions derived from observational studies to those in simulations and semi-analytic models at radii of order a few $\mathrm{kpc}$, as have been performed so far (e.g., Zentner \& Bullock 2003; Mao et al. 2004), do not constitute fair comparisons. This alone warrants further study of the effects of nonlocal perturbers.

What about nonlinear perturbers? In Figure 7, we show the expected number of non-linear perturbers $\left\langle N \mid \delta>\Delta_{\mathrm{NL}}\right\rangle$, for $\Delta_{\mathrm{NL}}=1$. It is clear that this number is quite small, typically $\langle N \mid \delta>1\rangle \lesssim 10^{-2}$. Even for a linear cutoff as small as $\Delta_{\mathrm{NL}}=20 \%$, the number of nonlinear perturbers is $\langle N| \delta>$ $20 \%\rangle \lesssim 10^{-1}$. The prevalence of nonlinear perturbers is unlikely, unless observational biases preferentially select systems 
where nonlinear effects are large, in which case more sophisticated methods must be used to compare theoretical predictions to observational data, perhaps using Monte Carlo methods like those of Zentner \& Bullock (2003) directly to model lens systems. While our results depend on our particular choice of profiles, it seems unlikely that they underestimate the number by significantly more than an order of magnitude, which is necessary to make nonlinear perturbers likely. Nevertheless, including many more massive substructures might add significantly to the number of nonlinear perturbers, as would adopting significantly higher substructure mass fractions, though higher mass fractions seem unlikely in the context of CDM.

Even in such a case, it is difficult to imagine that the number of nonlinear perturbers could be greater than a few. We would then expect the contribution to the magnification perturbation by nonlinear perturbers to have a large rms scatter so that $\delta_{\mathrm{T}}^{\mathrm{rms}} \sim\left\langle\delta_{\mathrm{T}}\right\rangle$. Because the scatter from linear perturbers appears to be broadly consistent with observed values, we expect it to be difficult to explain a nonzero net magnification perturbation with nonlinear perturbers without at the same time increasing the scatter in the perturbations beyond that of observations.

If neither nonlocality of the perturbations nor nonlinear perturbers can explain how positive- (negative-) parity images get brighter (dimmer) on average, then the only remaining possibility that is consistent with perturbations caused by CDM substructure is that for any given lens, the best-fit smooth model prediction for the magnification is a poor proxy for what the magnification would be if the mass in substructures were redistributed as a smooth component. We address these problems in detail in a forthcoming companion paper (Chen \& Rozo 2005, In preparation).

Before proceeding, we emphasize the importance of tidal truncation of substructures in the expected number of nonlinear perturbers. In particular, if we assume pure SIS perturber profiles, the number of perturbers in the region $|\delta|>20 \%$ increases by a factor of $\sim 10$ and becomes of order $\sim 1$, as was demonstrated by Chen et al. (2003b, compare to our Figure B8). This reflects the fact that, in our model, $\sigma_{\text {SIS }}(20 \%) \gtrsim a^{2}$ for substructures of mass $m_{\max }$, implying that substructures become tidally truncated within the region $\delta_{S I S}>20 \%$. Thus, use of simple SIS profiles that are not tidally-truncated may overestimate the importance of nonlinear substructures. Moreover, this implies that in order to draw robust conclusions from lens systems, the effective truncation of substructures must be well understood in systems with a variety of different formation histories, a problem which is theoretically challenging.

\subsection{Individual Lenses}

Though the model that we presented is simplified and our results should generally be interpreted on a statistical basis, it is interesting to consider how this analysis can aid in the understanding of individual systems. For instance, if the probability of finding a substructure within a tidal radius of an image is small, then the average perturbation ought to be a rather poor estimator of the most likely perturbation. In particular, the geometric weighting $\theta^{2}$ of substructures a distance $\theta$ away from the image is clearly not appropriate in this case. Conversely, a large number of perturbers within a tidal radius would allow us to invoke the central limit theorem, in which case the probability distribution $\rho\left(\delta_{\mathrm{T}}\right)$ would be well approximated by a gaussian distribution. This raises a natural question. How many perturbers are found within a tidal radius or, more pre- cisely, how many perturbers are found in the region $\delta>\delta_{\mathrm{p}}$ ? In what follows, we refer to these two conditions interchangeably. The rigorous condition will always be $\delta>\delta_{\mathrm{p}}$, and we loosely interpret this as perturbers found within a tidal radius from the image.

Figure 7 shows that for typical lens systems, the expected number of substructures with $\delta>\delta_{\mathrm{p}}$ is of order $\sim 20$ when all substructure parameters are as described in $\S 4.4$. The exact number is somewhat sensitive to the cutoff and density profile. For instance, at $\lambda_{a}=1$ the number of perturbers within a tidal radius decreases to a few to several perturbers, and increases again to $\sim 10$ perturbers if one lowers $m_{\max }$ by an order of magnitude. It seems likely then that in most lens systems the total magnification perturbation is due to from a few to about twenty substructures contributing at approximately comparable levels. Thus we expect that actual perturbations may differ substantially from the average perturbation, implying a large variance (see Figure 6) and a non-gaussian probability distribution for the magnification perturbations.

\section{DISCUSSION AND CONCLUSIONS}

The most surpising result from our analysis is that at a fixed lens mass, introducing a clumpy component tends to demagnify positive-parity images while magnifying negative-parity images on average, with the net effect tending towards zero as nonlinear perturbers become less important. This does not imply that the traditional arguments that adding substructure will, on average, increase the magnification of positive-parity images are wrong. Rather, it emphasizes that the increase in magnification of the observed image is, in part, due to the simple fact that mass is being added to the lens.

If one adopts the predicted magnification from the best-fit smooth macromodel to a lens as a proxy for what the magnification would have been if the substructure were not present and the lens were smooth, the observed average perturbation is positive, opposite from what we predict. For low substructure mass fractions (comparable to the level predicted by the CDM paradigm of structure formation), nonlinear perturbers are unlikely and hence nonlinearities, which were disregarded in our study, are not a likely explanation for the observed discrepancy. Further, if the scatter due to linear perturbers is found to broadly match that in observations, it will be difficult for nonlinear perturbers to explain the observed average perturbation without, at the same time, significantly increasing the scatter. This suggests that the use of the best-fit smooth model magnification as a proxy for the magnification of a smooth lens may not be justified. We investigate this specific question in more detail in a follow-up study (Chen \& Rozo 2005, in preparation).

Another surprising aspect of our analysis is the possibility of having sizable magnification perturbations arising from small, but coherent, contributions from several substructures within the lens halo. Such perturbations are difficult to account for in individual lenses because knowledge of the expected nongaussian distribution $\rho\left(\delta_{\mathrm{T}}\right)$ of the perturbations is necessary to perform maximum likelihood analyses of particular systems (e.g., Mao \& Schneider 1998). Likewise, the conclusions of analyses that assume a single substructure is responsible for the full magnification perturbation (e.g., Keeton 2001; Dobler \& Keeton 2005) can change when multiple perturbers are present. As a simple example, the relation between magnification perturbation cross section and the probability of an image being perturbed is weakened if large perturbations can arise from the 
coherent contribution of multiple, weak perturbations.

We emphasize that the importance of coherent perturbations depends on the tidal radii of substructures. This result underlines the importance of numerical studies such as those by Bradăc et al. (2004) and Amara et al. (2004), where tidal limitation of substructures is inherent in the calculation. Yet the theoretical challenges remain significant. The fact that we expect low-mass substructures (i.e. substructures with masses below the resolution limit of present day numerical simulations) to contribute to magnification perturbations at a non-negligible level and the fact that the scatter in the properties of substructure from halo to halo is significant both argue for complementing direct numerical simulation with analytic (e.g., Chen et al. 2003a) and semi-analytic computations (e.g., Zentner \& Bullock 2003; Zentner et al. 2005a; Taylor \& Babul 2005). Of course, the drawback of these studies is that substructure dynamics and tidal truncation are handled only in an approximate way.

One issue that was brought up in our analysis was the definition of the substructure mass fraction. In particular, we focused on magnification perturbations induced by granularity, so we argued that for a host halo with with a mass $M_{E}$ within its Einstein radius, one is interested in the mass fraction of substructures with mass $m \lesssim m_{\max }$, where $m_{\max } \approx 10^{-2} M_{E} \sim 10^{-4} M_{\mathrm{vir}}$. Unfortunately, the huge dynamic range that is necessary to probe the properties of halo substructure with $N$-body simulations limits their ability to probe substructures to masses larger than $m \gtrsim 10^{-5} M_{\text {vir }}$ (e.g., Mao et al. 2004) and to limit such studies to a relatively small number of systems. Therefore the CDM predictions for the appropriate substructure values require some extrapolation and are thus may be robust than one might hope. There are other theoretical uncertainties as well. In particular, Oguri (2004) pointed out that in systems where the lens galaxy is a member of a group or a cluster, the relevant substructure mass fraction should include contributions from substructures within the group halo itself. Likewise, anisotropy of the host halo and its substructure population (e.g., Zentner et al. 2005c; Kang et al. 2005; Libeskind et al. 2005) leads to significant systematic variations in substructure mass fractions along various lines of sight and may alter the expectation value of the substructure mass fraction due to projection effects (Zentner, Rozo, \& Kravtsov 2005, In preparation). Finally, the abundance of subhalos in CDM cosmologies depends on the amplitude of the power spectrum on small scales (Zentner \& Bullock 2003), and may depend as well on baryonic processes within the host halo that are yet to be fully understood. More theoretical work is needed to fully characterize the CDM predictions for the substructure mass fractions relevant for lensing.

While the detection of dark substructures through image splitting or magnification perturbations of extended sources (Inoue \& Chiba 2003), would provide fairly unambiguous evidence of CDM substructures, until such detections become feasible our only way to probe these dark substructures is through their effects on multiply-imaged sources. This makes unambiguous detection of dark substructures in individual lenses more difficult because more "mundane" causes for the smallscale perturbations to the lensing potentials exist. Spectroscopic analyses of lenses may go some way in removing these ambiguities (Moustakas \& Metcalf 2003), especially if complemented by studies of the associated astrometric perturbations (Chen et al. 2005, in preparation) and by looking for distortions in lensed jets (Metcalf \& Madau 2001). In light of these ambi- guities, we believe that if CDM were to provide concrete predictions for the statistical properties of the magnification perturbations induced by substructures, confirmation of these properties in observed lens samples would provide strong evidence in support of the CDM paradigm. In this paper, we have identified one way (granularity) in which CDM substructures can produce flux perturbations, and shown analytically that a proper understanding of the substructure profiles, including their tidal limitation, and the mass spectrum of subhalos are all necessary components of a faithful treatment of the magnification perturbations and to obtain accurate estimates of substructure abundance. Nevertheless, we also expect other effects such as perturbations by massive substructures (Cohn \& Kochanek 2004) to play important roles, though the corresponding statistical properties of these perturbations remain to be understood. While a complete of understanding of the CDM predictions for the statistical properties of magnification perturbations is a difficult task, it is one which is likely to be of vital importance for testing the CDM paradigm at small scales and in the highlynonlinear regime.

We would like to thank Scott Dodelson for useful discussions throughout the development of this work, and Charles Keeton for a carfeul reading and critique of this manuscript. We also thank Andrey Kravtsov for valuable comments on the manuscript. ER, ARZ, and JC are supported by The Kavli Institute for Cosmological Physics at The University of Chicago and by the National Science Foundation under grant NSF PHY 0114422. GB is supported by the DOE and the NASA grant NAG 5-10842 at Fermilab. This research made use of the NASA Astrophysics Data System.

\section{APPENDIX}

\section{A. WHEN CAN ASTROMETRIC PERTURBATIONS BE NEGLECTED?}

In general, if the potential of a macrolens is perturbed, the image position of a source will change in accordance with the perturbed lens equation. We wish to determine under what conditions is it possible to neglect such perturbations in comparison to the magnification perturbations.

Consider a lens system where the unperturbed image position of a point source is $\boldsymbol{\theta}$. Neglecting astrometric perturbations, the only change to the convergence and shear sampled by the image are the values $\delta \kappa$ and $\delta \gamma$ of the perturber at the image position. Relative to this first-order perturbation, there are higher order corrections due to the image being displaced because both the macromodel and perturber convergence and shear at the new image position will be different. For these higher order terms to be negligible, it is required that

$$
\delta \kappa \gg \frac{\partial(\delta \kappa)}{\partial \boldsymbol{\theta}} \delta \boldsymbol{\theta} \text { and } \delta \gamma \gg \frac{\partial \gamma}{\partial \boldsymbol{\theta}} \delta \boldsymbol{\theta}
$$

where $\delta \boldsymbol{\theta}$ is the position perturbation, and similar inequalities must hold for the image shear. To determine when these conditions are met, one needs to compute the perturbation $\delta \boldsymbol{\theta}$.

Let a spherically-symmetric perturber be introduced at a position $\boldsymbol{\theta}_{\mathrm{p}}$. The perturbed lens equation is

$$
\boldsymbol{\theta}+\delta \boldsymbol{\theta}=\boldsymbol{\beta}+\boldsymbol{\alpha}(\boldsymbol{\theta}+\delta \boldsymbol{\theta})+\delta \boldsymbol{\alpha}(\boldsymbol{\theta}+\delta \boldsymbol{\theta}) .
$$

$\delta \boldsymbol{\alpha}$ is the deflection due to the perturber, which is a function of the relative position of the perturber $\boldsymbol{\theta}+\delta \boldsymbol{\theta}-\boldsymbol{\theta}_{\mathrm{p}}$. Linearizing $\boldsymbol{\alpha}$ and using the unperturbed lens equation $\boldsymbol{\theta}=\boldsymbol{\beta}+\boldsymbol{\alpha}(\boldsymbol{\theta})$, we obtain

$$
M^{-1} \delta \boldsymbol{\theta}=\delta \boldsymbol{\alpha}(\boldsymbol{\theta}+\delta \boldsymbol{\theta})
$$


where $M^{-1}$ is the inverse magnification tensor due to the macromodel, $M^{-1}{ }_{i j}=\delta_{i j}-\partial \alpha_{i} / \partial \theta_{j}$. To get this equation we only needed to assume that the macrolens properties vary slowly over a distance $|\delta \theta|$. To fully linearize the system and set $\delta \boldsymbol{\alpha}(\boldsymbol{\theta}+\delta \boldsymbol{\theta}) \approx \delta \boldsymbol{\alpha}(\boldsymbol{\theta})$, the system must satisfy the more stringent constraint

$$
|\delta \boldsymbol{\alpha}| \gg\left|\frac{\partial \delta \boldsymbol{\alpha}}{\partial \boldsymbol{\theta}} \delta \boldsymbol{\theta}\right|
$$

Assuming we can fully linearize the system and inverting we obtain

$$
\delta \boldsymbol{\theta}=M \delta \boldsymbol{\alpha} .
$$

However, there is a self-consistency constraint provided by Equation A4 that must be satisfied. Inserting the fullylinearized solution into Equation A4, we find that one must have

$$
|\delta \boldsymbol{\alpha}| \gg\left|\frac{\partial \delta \boldsymbol{\alpha}}{\partial \boldsymbol{\theta}} M \delta \boldsymbol{\alpha}\right| .
$$

Consider the matrix

$$
\frac{\partial \delta \boldsymbol{\alpha}}{\partial \boldsymbol{\theta}} M=\left(\begin{array}{cc}
\delta \kappa+\delta \gamma_{1} & \delta \gamma_{2} \\
\delta \gamma_{2} & \delta \kappa-\delta \gamma_{1}
\end{array}\right)\left(\begin{array}{cc}
1 / \lambda_{T} & 0 \\
0 & 1 / \lambda_{R}
\end{array}\right)
$$

where $\lambda_{R}=1-\kappa+\gamma$ and $\lambda_{T}=1-\kappa-\gamma$. Typically, one is interested in images that form near the tangential critical curve of the macrolens, which satisfy $1 / \lambda_{T} \gg 1 / \lambda_{R}$. Setting $1 / \lambda_{R}=0$ in the above expression leads to

$$
\frac{\partial \delta \boldsymbol{\alpha}}{\partial \boldsymbol{\theta}} M \approx 1 / \lambda_{T}\left(\begin{array}{cc}
\delta \kappa+\delta \gamma_{1} & 0 \\
\delta \gamma_{2} & 0
\end{array}\right)
$$

The eigenvalues of this matrix are zero and $\left(\delta \kappa+\delta \gamma_{1}\right) / \lambda_{T}$. Letting $\mathbf{e}$ be the unit eigenvector corresponding to the nonzero eigenvalue, it follows that

$$
\left|\frac{\partial \delta \boldsymbol{\alpha}}{\partial \boldsymbol{\theta}} \boldsymbol{M} \delta \boldsymbol{\alpha}\right| \approx \frac{1}{\lambda_{T}}\left(\delta \kappa+\delta \gamma_{1}\right)(\mathbf{e} \cdot \delta \boldsymbol{\alpha})<\frac{1}{\lambda_{T}}(\delta \kappa+\delta \gamma)|\delta \boldsymbol{\alpha}| .
$$

Inserting this result back into Equation (A6), we find that full linearization of the problem is self consistent when

$$
\frac{1}{\lambda_{T}}(\delta \kappa+\delta \gamma) \ll 1
$$

Now that we have found a consistent way to estimate the astrometric perturbation to the image position, we can estimate the change in macromodel and perturber convergences between the original, unperturbed image position and the new image position.

Consider first the macromodel. If $\theta_{E}$ is the Einstein radius of the macromodel, one typically expects $\partial \kappa / \partial \theta \approx \kappa / \theta_{E}$ for the macromodel, and $\delta \alpha \approx \delta \kappa \theta$ for the deflection angle due to the perturber. Inserting these into Eq. (A1) and using $\delta \theta \approx M \delta \alpha \approx$ $\lambda_{T} \delta \kappa \theta$, we find that position perturbations are negligible as far as the macromodel is concerned provided

$$
\theta \ll \lambda_{T} \theta_{E} \lesssim 0.1 \theta_{E} .
$$

That is, position perturbations due to nearby perturbers are expected to be negligible (relative to the convergence perturbation $\delta \kappa)$. The corresponding condition for the perturber is

$$
\frac{\delta \kappa}{\lambda_{T}} \ll 1,
$$

a constraint that is already contained within the self-consistency requirement given by Eq. (A10). A similar computation for the shear yields the exact same conclusions. It follows that if the linearization condition, Eq. (A10), holds, we may neglect astrometric perturbations when computing magnification perturbations.
We emphasize that if an image lies very close to the tangential critical curve, the condition expressed in Eq. (A10) may represent a very stringent requirement. For order of magnitude purposes, one can assume $\delta \kappa \sim \delta \gamma$ and $\lambda_{R} \sim 1$, so that our requirement for neglecting astrometric perturbations can be roughly expressed as

$$
\delta \kappa+\delta \gamma \ll 1 /|\mu| \text {. }
$$

In particular, this means that the maximum value that a perturbation $\delta \kappa$, or $\delta \gamma$, can take while still producing negligible astrometric perturbations is of order $|\mu|^{-1}$. It is possible to go even further and relate this to flux perturbations. We saw in Eq. (2) that for small perturbations $\delta \kappa$ and $\delta \gamma$, the flux perturbation is given by

$$
\frac{\delta \mu}{|\mu|}=2|\mu|\left\{(1-\kappa) \delta \kappa+\gamma \delta \gamma_{1}\right\} .
$$

One expects in general that $\kappa \sim \gamma \sim 1 / 2$, so that the relative flux perturbation $\delta \mu /|\mu|$ is of order

$$
\frac{\delta \mu}{|\mu|} \sim|\mu|(\delta \kappa+\delta \gamma)
$$

The linearization condition we derived, Equation (A13), states that the right-hand side of Eq. (A15) ought to be much smaller than one. Hence, another way of phrasing the linearization condition is simply

$$
\frac{\delta \mu}{|\mu|} \ll 1
$$

That is, astrometric perturbations to the image position may be neglected when considering small flux perturbations.

\section{B. POINT MASS PERTURBERS}

Consider the perturbations due to a point mass of mass $m$. The perturbing potential, convergence, and shear are

$$
\delta \psi(\theta)=b^{2} \ln (\theta), \quad \delta \kappa(\theta)=0, \quad \text { and } \delta \gamma(\theta)=\frac{b^{2}}{\theta^{2}},
$$

where $b^{2}=m / \pi \Sigma_{\mathrm{c}} D_{\mathrm{L}}^{2}$ is the Einstein radius of the point mass. Inserting these expressions in Equations (4), we find only one root so that

$$
\theta_{\max }^{2}(\Delta)=\frac{2|\mu| \gamma}{|\Delta|} b^{2} \quad \text { and } \quad \theta_{\min }^{2}(\Delta)=0
$$

for both $\Delta>0$ and $\Delta<0$. Likewise, inserting the shear and convergence profiles in Equation 5, we find

$$
2 \phi_{\mathrm{m}}(\Delta \mid \theta)=\cos ^{-1}\left(-\frac{\Delta}{|\Delta|} \frac{\theta^{2}}{\theta_{\max }^{2}}\right) .
$$

Using the above expressions and carrying out the integrals in Equation (6), we find

$$
\sigma(\Delta)=\frac{2 \gamma|\mu|}{|\Delta|} b^{2} .
$$

This holds for both $\Delta>0$ and $\Delta<0$; the cross sections for brightening and dimming an image are exactly equal to each other. This is to be expected: point mass perturbers couple only through the shear, so perturbers placed at angles $\phi$ and $\phi+\pi / 2$ create equal and opposite perturbations. Since no such symmetry exists for perturbers with nonzero convergence, the equality $\sigma(\Delta)=\sigma(-\Delta)$ does not hold in general. This symmetry also implies that $y_{1}(\delta)=0$ and hence the average magnification perturbation due to point masses is zero ${ }^{12}$. However, the variance

12 This is not correct when nonlinear perturbations are likely, in which case negative-parity images have a large probability of being strongly demagnified. Positive-parity images are also demagnified on average, but the effect is not as strong as for negative-parity images (Schechter \& Wambsganss 2002). 


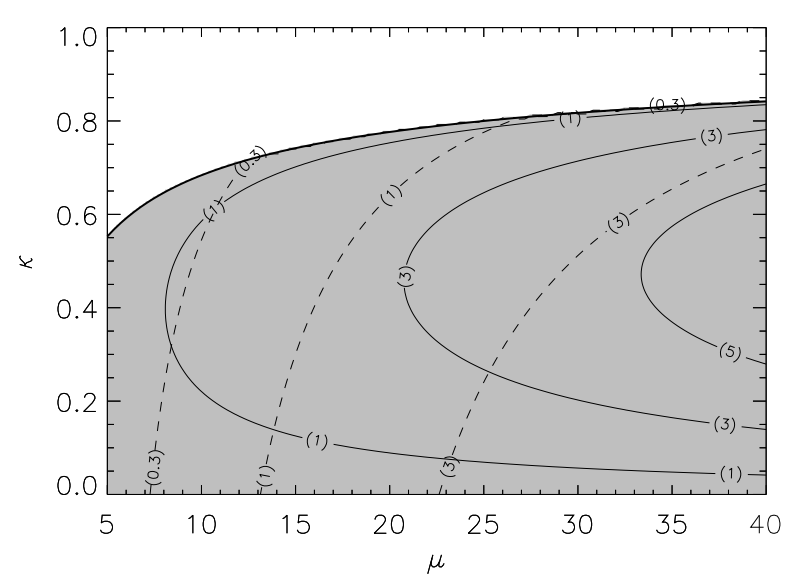

FIG. B 8.- The solid contours give the expected number of stars in a lensing galaxy which produce strong (nonlinear) magnification perturbations on an image. A linear cutoff $\Delta_{\mathrm{NL}}=0.2$ and a stellar mass fraction $f_{*}=0.1$ at the galaxy's Einstein radius are assumed. The lightly-shaded area represents the allowed region of parameter space for positive-parity images, and contours are for 1,3 , and 5 nonlinear stars. We conclude that stellar magnification perturbations to multiply-imaged quasars will generally be large and nonlinear (hence microlensing). The dashed contours illustrate the number of mildly nonlinear $(\delta>20 \%)$ SIS perturbers predicted by an SIS model assuming that the number density of perturbers is equal to that assumed in $\S 4.4$ for pseudo-Jaffe profiles. This is to be compared to the dashed lines in Fig. 7. The number of nonlinear SIS perturbers is of order unity, whereas the number of nonlinear perturbers for the truncated profiles is $\lesssim 0.1$. This reflects the fact that tidal truncation occurs within the region $\delta>20 \%$ for an SIS perturber. We conclude that SIS profiles lead to strong overestimates of the importance of nonlinear perturbers.

is nonzero. Inserting Eq. (B4) into Eq. (14), and using the definition of the Einstein radius $\pi \Sigma_{\mathrm{c}} D_{\mathrm{L}}^{2} b^{2}=m$, we obtain

$$
\begin{aligned}
\operatorname{VAR}\left(\delta_{\mathrm{T}}\right) & =\int d m \frac{1}{\Sigma_{\mathrm{c}}} \frac{d s}{d m} m \frac{4}{\pi} \gamma|\mu| \Delta \\
& =\frac{4}{\pi} \gamma \kappa|\mu| \Delta f_{\text {sub }}=\langle N \mid \Delta\rangle \Delta^{2},
\end{aligned}
$$

where we have defined the perturber mass fraction $f_{\text {sub }}$, such that $f_{\text {sub }} \kappa \Sigma_{\mathrm{c}}=\int d m \frac{d s}{d m} m$ and $\langle N \mid \Delta\rangle$ is the expected number of perturbers that produce a perturbation stronger than $\Delta$. Interestingly, the variance of the perturbation does not depend in any way on the perturber mass spectrum.

Lensing by stars: As a particular application, consider magnification perturbations due to stars in the lens galaxy. We take all stars to be identical in mass and define $f_{*}$ as the surface mass fraction of stars at the image position, $f_{*}=\Sigma_{*} / \Sigma_{\text {Tot }}$. The expected number of stars in the region $|\delta|>\Delta$ (note the absolute value) is thus

$$
\langle N|| \delta \mid>\Delta\rangle=\frac{f_{*} \kappa}{m_{*}} D_{\mathrm{L}}^{2}\{\sigma(\Delta)+\sigma(-\Delta)\}=\frac{4}{\pi} \frac{|\mu| \gamma \kappa}{\Delta} f_{*}
$$

For $f_{*} \gtrsim 0.1, \kappa \approx \gamma \approx 1 / 2$, and $\mu \approx 10$, we get $\langle N|| \delta|>$ $1\rangle \gtrsim 0.3$. We expect then, that nonlinear perturbers will play an important role in determining the observed magnification of an image, a result that has been amply demonstrated in previous studies (see for example Schechter \& Wambsganss 2002)

\section{SINGULAR ISOTHERMAL SPHERE PERTURBERS}

Consider perturbations by a singular isothermal sphere. The perturbing potential, convergence, and shear profiles are

$$
\delta \psi(\theta)=b \theta, \quad \delta \kappa=\frac{b}{2 \theta}, \quad \text { and } \delta \gamma=\frac{b}{2 \theta},
$$

where $b$ is the Einstein radius of the perturber, given by

$$
b=4 \pi\left(\frac{\sigma_{v}}{c}\right)^{2} \frac{D_{\mathrm{LS}}}{D_{\mathrm{S}}},
$$

and $\sigma_{v}$ is the velocity dispersion of the perturber. From Equation (4), the nonzero solutions for $\theta_{\max }$ are

$$
\theta_{\max }(\Delta)=\frac{|\mu| b}{|\Delta|} \times \begin{cases}\lambda_{R} & \text { if } \Delta>0 \text { and } \lambda_{R} \geq 0 \\ -\lambda_{T} & \text { if } \Delta<0 \text { and } \lambda_{T} \leq 0 .\end{cases}
$$

$\theta_{\max }=0$ for $\Delta>0, \lambda_{+}<0$ and for $\Delta<0, \lambda_{-}>0$, and $\theta_{\min }=0$ always. Note $\theta_{\max }(\Delta)=0$ implies that perturbations of size $\Delta$ are never realized, so it is impossible for SIS perturbers to either dim a positive-parity image or brighten a doubly negative-parity image. Finally, from Equation (5), we find

$$
2 \phi_{m}(\theta \mid \Delta)=\cos ^{-1}\left(\frac{1-\kappa}{\gamma}-\frac{1}{\gamma|\mu|} \frac{\theta}{b} \Delta\right) .
$$

Inserting our above expressions into Equation (6), and defining the amplitude $\sigma_{0}$ via

$$
\sigma_{0}=\frac{\pi}{2} \frac{|\mu|^{2} b^{2}}{\Delta^{2}}
$$

we obtain

- $\Delta>0, \lambda_{-}>0, \lambda_{+}>0$ : Brightening of a positive-parity image.

$$
\sigma=\sigma_{0}\left[2(1-\kappa)^{2}+\gamma^{2}\right]
$$

- $\Delta>0, \lambda_{-}<0, \lambda_{+}>0$ : Dimming of a negative-parity image. ${ }^{13}$

$$
\sigma=\sigma_{0}\left\{\frac{1}{\pi} \cos ^{-1}\left(-\frac{|1-\kappa|}{\gamma}\right)\left[2(1-\kappa)^{2}+\gamma^{2}\right]+\frac{3}{\pi} \frac{1-\kappa}{|\mu|^{1 / 2}}\right\}
$$

- $\Delta<0, \lambda_{-}<0, \lambda_{+}>0$ : Brightening of a negative-parity image.

$$
\sigma=\sigma_{0}\left\{\frac{1}{\pi} \cos ^{-1}\left(\frac{|1-\kappa|}{\gamma}\right)\left[2(1-\kappa)^{2}+\gamma^{2}\right]-\frac{3}{\pi} \frac{1-\kappa}{|\mu|^{1 / 2}}\right\}
$$

- $\Delta<0, \lambda_{-}<0, \lambda_{+}<0$ : Dimming of a doubly negativeparity image.

$$
\sigma=\sigma_{0}\left[2(1-\kappa)^{2}+\gamma^{2}\right]
$$

The expressions above are in exact agreement with the results from Keeton (2003) in the limit $|\Delta| \ll 1$. In particular, the cross sections in Keeton (2003) contain terms that are higher order in $\Delta$, not found in our expressions. However, as we already noted, these corrections are important only when astrometric perturbations become non-negligible. Since negligible astrometric perturbations were also assumed in Keeton (2003), these higher-order terms are important only when both formalisms break down.

Before moving on, it is worth noticing that while the cross sections we have derived depend on the parity of the image, this dependence is something of happenstance. In particular, what differentiates the various expressions for the magnification perturbation cross sections is not the image parity, but whether Equation (4) has zero, one, or two roots. For an SIS

13 Even though the result we quote here looks quite different from the one presented in Equation 17 of Keeton (2003), it is simple to show that $\pi-2 \theta_{\delta}=$ $\cos ^{-1}\left(-\frac{|1-\kappa|}{\gamma}\right)$, so that both expressions for $\sigma(\Delta)$ do indeed agree to leading order in $\delta\left(\theta_{\delta}\right.$ is defined in Keeton (2003)). A similar argument holds for the case $\Delta<0, \lambda_{T}<0, \lambda_{R}>0$. 
perturber, these conditions coincide with the parity of the image, but this is not true in general. Indeed, we already showed in Appendix B, that point masses do not distinguish between positive- and negative-parity images.

Consider an ensemble of SIS perturbers, which we choose to parameterize through their Einstein radii $b$. Assuming the macroimage has positive parity, Equation (13) becomes

$$
\left\langle\delta_{\mathrm{T}}\right\rangle=\pi|\mu|^{2}\left[2(1-\kappa)^{2}+\gamma^{2}\right] \int d b \frac{d s}{d b} b^{2}\left[1 / \delta_{m}-1 / \Delta\right],
$$

which clearly diverges in the limit $\delta_{m} \rightarrow 0$. The physical reason behind this is clear: each individual perturber within a distance $\theta$ from the image contributes a positive perturbation $\delta \sim 1 / \theta$. Since the number of perturbers in such a ring scales as $\theta$, the net perturbation is divergent.

How generic are these divergences? If the magnification perturbation is dominated by the convergence perturbation $\delta \kappa$, then perturbers along a ring will give nonzero average perturbations that scale as $\theta \delta \kappa$. Convergence is expected then if, and only if, $\delta \kappa$ falls faster than $1 / \theta^{2}$. Since any such perturber has a finite mass, we conclude that finite magnification perturbations are expected if and only if the perturbers have finite masses.

\section{REFERENCES}

Amara, A., Metcalf, R. B., Cox, T. J., \& Ostriker, J. P. 2004, astro-ph/0411587 Baltz, E. A., Briot, C., Salati, P., Taillet, R., \& Silk, J. 2000, PRD, 61, 023514

Benson, A. J., Frenk, C. S., Lacey, C. G., Baugh, C. M., \& Cole, S. 2002, MNRAS, 333, 177

Berezinsky, V., Bottino, A., \& Mignola, G. 1997, Physics Letters B, 391, 355

Bergström, L., Edsjö, J., Gondolo, P., \& Ullio, P. 1999, PRD, 59, 043506

Bertone, G., Hooper, D., \& Silk, J. 2005, Phys. Rept., 405, 279

Blumenthal, G. R., Faber, S. M., Primack, J. R., \& Rees, M. J. 1984, Nature, 311,517

Bode, P., Ostriker, J. P., \& Turok, N. 2001, ApJ, 379, 440

Bottino, A., Donato, F., Fornengo, N., \& Salati, P. 1998, PRD, 58, 123503

Bradăc, M. 2002, A \& A, 388, 373

Bradăc, M., Schneider, P., Lombardi, M., Steinmetz, M., Koopmans, L. V. E., \& Navarro, J. F. 2004, A \& A, 423, 797

Bullock, J. S., Kravtsov, A. V., \& Weinberg, D. H. 2000, ApJ, 539, 517

Calcáneo-Roldán, C. \& Moore, B. 2000, PRD, 62, 123005

Chen, D. N., Jing, Y. P., \& Yoshikaw, K. 2003a, ApJ, 597, 35

Chen, J., Kravtsov, A. V., \& Keeton, C. R. 2003b, ApJ, 592, 24

Chiba, M. 2002, ApJ, 565, 17

Cohn, J. D. \& Kochanek, C. S. 2004, ApJ, 608, 25

Cole, S., Aragon-Salamanca, A., Frenk, C. S., Navarros, J. F., \& Zepf, S. E. 1994, MNRAS, 271, 781

Colín, P., Avila-Reese, V., \& Valenzuela, O. 2000, ApJ, 542, 622

Dalal, N. \& Kochanek, C. S. 2002a, Apj, 572, 52

-. 2002b, astro-ph/0202229

De Lucia, G., Kauffmann, G., Springel, V., White, S. D. M., Lanzoni, B.,

Stoehr, F., Tormen, G., \& Yoshida, N. 2004, MNRAS, 348, 333

Dekel, A. \& Silk, J. 1986, ApJ, 303, 38

Diemand, J., Moore, B., \& Stadel, J. 2004, MNRAS, 352, 535

Dobler, G. \& Keeton, C. R. 2005, ApJ, Submitted (astro-ph/0502436)

Donato, F., Fornengo, N., Maurin, D., \& Salati, P. 2004, Phys. Rev., D69, 063501

Efstathiou, G. 1992, MNRAS, 256, 43P

Evans, N. W. \& Witt, H. J. 2003, MNRAS, 245, 1351

Gao, L., White, S. D. M., Jenkins, A., Stoehr, F., \& Springel, V. 2004, MNRAS, accepted

Gaudi, B. S. \& Petters, A. O. 2002, ApJ, 580, 468

Ghigna, S., Moore, B., Governato, F., Lake, G., Quinn, T., \& Stadel, J. 2000, ApJ, 544, 616

Hayashi, D., Navarro, J. F., Taylor, J. E., Stadel, J., \& Quinn, T. 2003, ApJ, 584,541

Hogan, C. J. \& Dalcanton, J. J. 2000, PRD, 62, 063511

Inoue, K. T. \& Chiba, M. 2003, ApJ, 591, L83

Kamionkowski, M. \& Liddle, A. R. 2000, PRL, 84, 4525

Kang, X., Mao, S., Gao, L., \& Jing, Y. P. 2005, Astron. \& Astrophys. Submitted (astro-ph/0501333)

Kauffmann, G., White, S. D. M., \& Guiderdoni, B. 1993, MNRAS, 264, 201

Kawano, Y. 2004, PASJ, 56, 253
Kazantzidis, S., Mayer, L., Mastropietro, C., Diemand, J., Stadel, J., \& Moore, B. 2004b, ApJ, 608, 663

Keeton, C. R. 2001, astro-ph/0111595

Keeton, C. R. 2003, ApJ, 587, 143

Keeton, C. R., Gaudi, B. S., \& Petters, A. . 2005, astro-ph/0503452

Keeton, C. R., Gaudi, B. S. \& Petters, A. O. 2003, ApJ, 598, 138

Keeton, C. R. \& Zabludoff, A. I. 2004, ApJ, 612, 660

Kembhavi, A. K. \& Narlikar, J. V. 1999, Quasars and active galactic nuclei : an introduction (Quasars and active galactic nuclei : an introduction /Ajit K. Kembhavi, Jayant V. Narlikar. Cambridge, U.K. : Cambridge University Press, c1999. ISBN 0521474779.)

Klypin, A. A., Kravtsov, A. V., Valenzuela, O., \& Prada, F. 1999, ApJ, 522, 82

Knebe, A., Devriendt, J. E. G., Mahmood, A., \& Silk, J. 2002, MNRAS, 329, 813

Kochanek, C. S. \& Dalal, N. 2004, ApJ, 610, 69

Koopmans, L. V. E. 2003, ApJ, 595, 712

Koushiappas, S. M., Zentner, A. R., \& Walker, T. P. 2004, Phys. Rev. D, 69, 043501

Kravtsov, A. V., Gnedin, O. Y., \& Klypin, A. A. 2004, ApJ, 609, 482 (KGK04)

Libeskind, N. I., Frenk, C. S., Cole, S., Helly, J. C., Jenkins, A., Navarro, J. F., \& Power, C. 2005, MNRAS, submitted (astro-ph/0503400)

Lin, W. B., Huang, D. H., Zhang, X., \& Brandenberger, R. 2001, PRL, 86, 954

Macció, A. V., Moore, B., Stadel, J., \& Diemand, J. 2005, astro-ph/0506125

Mao, S., Jing, Y., Ostriker, J. P., \& Weller, J. 2004, ApJ, 604, L5

Mao, S. \& Schneider, P. 1998, MNRAS, 295, 587

McGaugh, S. S., Barker, M. K., \& de Blok, W. J. G. 2003, ApJ, 584, 566

Metcalf, R. B. 2004, ApJ, 607, 43

-. 2004a, astro-ph/0407298

-. 2004b, astro-ph/0412538

Metcalf, R. B. \& Madau, P. 2001, ApJ, 563, 9

Metcalf, R. B. \& Zhao, H.-S. 2002, ApJL, 567, L5

Möller, O. 2002, ApJ, 573, 562

Möller, O. \& Blain, A. W. 2001, MNRAS, 327, 339

Möller, O., Hewett, P., \& Blain, A. W. 2003, MNRAS, 245, 1

Moore, B., Ghigna, S., Governato, F., Lake, G., Quinn, T., Stadel, J., \& Tozzi, P. 1999, ApJL, 524, L9

Moustakas, L. A. \& Metcalf, R. B. 2003, MNRAS, 229, 607

Muñoz, J. A., Kochanek, C. S., \& Keeton, C. R. 2001, ApJ, 558, 657

Nagai, D. \& Kravtsov, A. V. 2005, ApJ, 618, 557

Oguri, M. 2004, astro-ph/0411464

Quadri, R., Möller, O., \& Natarajan, P. 2003, ApJ, 597, 659

Rees, M. J. 1986, MNRAS, 218, 25P

Refsdal, S. 2000, A \& A, 360

Richards, G. T. 2004, ApJ, 610, 679

Schechter, P. L. 2003, ApJ, 584, 657

Schechter, P. L. \& Wambsganss, J. 2002, ApJ, 580, 685

Schechter, P. L., Wambsganss, J., \& Lewis, G. F. 2004, ApJ, 613, 77

Schild, R. E. 1996, ApJ, 464, 125

Schneider, P., Ehlers, J., \& Falco, E. E. 1992, Gravitational Lenses (Gravitational Lenses, XIV, 560 pp. 112 figs.. Springer-Verlag Berlin Heidelberg New York. Also Astronomy and Astrophysics Library)

Schneider, P. \& Weiss, A. 1992, A \& A, 260, 1

Shapiro, P. R., Giroux, M. L., \& Babul, A. 1994, ApJ, 427, 25

Sigurdson, K. \& Kamionkowski, M. 2004, Physical Review Letters, 92, 171302

Silk, J. \& Stebbins, A. 1993, Astrophys. J., 411, 439

Somerville, R. S. 2002, ApJ, 572, 23

Somerville, R. S. \& Primack, J. R. 1999, MNRAS, 310, 1087

Spergel, D. N. \& Steinhardt, P. J. 2000, PRL, 84, 3760

Stoehr, F., White, S. D. M., Springel, V., Tormen, G., \& Yoshida, N. 2003, MNRAS, 345, 1313

Stoehr, F., White, S. D. M., Tormen, G., \& Springel, V. 2002, MNRAS, 335, L84

Tasitsiomi, A. \& Olinto, A. V. 2002, PRD, 66, 083006

Taylor, J. E. \& Babul, A. 2005, MNRAS, Submitted

Thoul, A. A. \& Weinberg, D. H. 1996, ApJ, 465, 608

van den Bosch, F. C., Mo, H. J., \& Yang, X. 2003, MNRAS, 345, 923

Wambsganss, J., Bode, P., \& Ostriker, J. P. 2004, ApJ submitted (astro$\mathrm{ph} / 0405147)$

White, S. D. M. \& Rees, M. J. 1978, MNRAS, 999, 999

Wisotzki, A. 2003, A \& A, 408, 455

Wozniak, P. R. 2000, ApJ, 529, 88

Yoo, J., Kochanek, C. S., Falco, E. E., \& McLeod, B. A. 2005, ApJ, 626, 51

Zakharov, A. F. 1995, A \& A, 293, 1

Zentner, A. R., Berlind, A. A., Bullock, J. S., Kravtsov, A. V., \& Wechsler, R. H. 2005a, ApJ, 624, 505

Zentner, A. R. \& Bullock, J. S. 2002, PRD, 66, 043003

-. 2003, ApJ, 598, 49

Zentner, A. R., Koushiappas, S. M., \& Kazantzidis, S. 2005b, in Proceedings of the Fifth International Workshop on the Identification of Dark Matter

Zentner, A. R., Kravtsov, A. V., Gnedin, O. Y., \& Klypin, A. A. 2005c, ApJ, In Press, (astro-ph/0502496) 